\title{
Isogeometric Reissner-Mindlin shell analysis with exactly calculated director vectors
}

\author{
W. Dornisch ${ }^{\mathrm{a}, *}$, S. Klinkel ${ }^{\mathrm{a}}$, B. Simeon ${ }^{\mathrm{b}}$ \\ ${ }^{a}$ Fachgebiet Statik und Dynamik der Tragwerke, Technische Universität Kaiserslautern, Paul-Ehrlich-Straße, 67663 Kaiserslautern, Germany \\ ${ }^{b}$ Arbeitsgruppe Differential-Algebraische Systeme, Technische Universität Kaiserslautern, Paul-Ehrlich-Straße, 67663 Kaiserslautern, Germany
}

\begin{abstract}
An isogeometric Reissner-Mindlin shell derived from the continuum theory is presented. The geometry is described by NURBS surfaces. The kinematic description of the employed shell theory requires the interpolation of the director vector and of a local basis system. Hence, the definition of nodal basis systems at the control points is necessary for the proposed formulation. The control points are in general not located on the shell reference surface and thus, several choices for the nodal values are possible. The proposed new method uses the higher continuity of the geometrical description to calculate nodal basis system and director vectors which lead to geometrical exact interpolated values thereof. Thus, the initial director vector coincides with the normal vector even for the coarsest mesh. In addition to that a more accurate interpolation of the current director and its variation is proposed. Instead of the interpolation of nodal director vectors the new approach interpolates nodal rotations. Account is taken for the discrepancy between interpolated basis systems and the individual nodal basis systems with an additional transformation. The exact evaluation of the initial director vector along with the interpolation of the nodal rotations lead to a shell formulation which yields precise results even for coarse meshes. The convergence behavior is shown to be correct for $k$-refinement allowing the use of coarse meshes with high orders of NURBS basis functions. This is potentially advantageous for applications with high numerical effort per integration point. The geometrically nonlinear formulation accounts for large rotations. The consistent tangent matrix is derived. Various standard benchmark examples show the superior accuracy of the presented shell formulation. A new benchmark designed to test the convergence behavior for free form surfaces is presented. Despite the higher numerical effort per integration point the improved accuracy yields considerable savings in computation cost for a predefined error bound.
\end{abstract}

Keywords: Isogeometric Analysis, Reissner-Mindlin Shell, NURBS, Interpolation of the Director

\section{Introduction}

The two main theories in shell analysis for engineering applications are the Kirchhoff-Love and the ReissnerMindlin shell theory. The shear-deformable Reissner-Mindlin shell theory has to be used to describe thick shells. While the Reissner-Mindlin shell theory can also be applied to thin shells, the Kirchhoff-Love theory is limited to the latter.

Kirchhoff-Love shells require $C^{1}$ continuity which is difficult to achieve for standard finite elements based on Lagrange shape functions. NURBS surfaces offer this continuity throughout a whole patch, which allows the implementation of isogeometric Kirchhoff-Love shells, see [1]. But the lack of rotational degrees of freedom entails

${ }^{*}$ Correspondence to: W. Dornisch, Lehrstuhl für Technische Mechanik, Technische Universität Kaiserslautern, Gottlieb-Daimler-Str., 67663 Kaiserslautern, Germany. E-mail: dornisch@rhrk.uni-kl.de

This is the peer reviewed version of the following article: Dornisch, W, Klinkel, S, and Simeon, B (2013): Isogeometric ReissnerMindlin shell analysis with exactly calculated director vectors. Comput. Methods Appl. Mech. Engrg. 253, 491-504, which has been published in final form at doi: 10.1016/j.cma.2012.09.010. (C2012. This manuscript version is made available under the CC-BY-NC-ND 4.0 license http://creativecommons.org/licenses/by-nc-nd/4.0/. 
problems regarding the imposition of boundary conditions. The connection of multiple patches requires additional efforts as $C^{0}$ continuity prevails at the border of patches [2].

The various locking phenomena occurring in Reissner-Mindlin shell analysis when applied to thin shells almost vanish when the order of the NURBS basis functions is elevated [3]. This renders Reissner-Mindlin shells more flexible in comparison to Kirchhoff-Love shells and opens a wide field of applications. The imposition of boundary conditions and the connection of multiple patches is straight-forward for Reissner-Mindlin shells. An isogeometric implementation based on a degenerated solid is described in [4].

Isogeometric analysis based on NURBS surfaces offers $C^{p-1}$ continuity throughout the element, which guaranties at least $C^{1}$ continuity for curved domains. This higher continuity allows a continuous determination of the normal vector. In contrast to that standard finite elements do not offer this possibility and thus have to rely on discrete predefined nodal director vectors. Depending on the interpolation strategy for rotations discrete nodal values of directors might also be needed in isogeometric Reissner-Mindlin shell analysis. In order to avoid any negative impact on the quality of the results these nodal values have to be chosen in a way, that the interpolated initial director vectors exactly coincide with the normal vectors derived from the geometry. In [5] additional factors computed on the element level to attain an exact interpolation of the normal vectors are proposed to overcome this issue. However this rotationfree shell is based on a discrete Kirchhoff formulation and thus neglects transverse shear deformations.

In this work we present a method to calculate exact nodal director vectors and nodal basis systems. This is done in a preprocess with a global system of equations and allows to use the unaltered NURBS basis functions for the interpolation of the director vector and the rotations. The proposed nonlinear Reissner-Mindlin shell formulation is derived from the continuum theory as proposed in [6]. It comprises a more accurate approach for the interpolation of the current director vector. The introduced interpolation maintains the orthogonality of the reference director vector and ensures the inextensibility of the shell. A new approach for the approximation of the variation of the director vector is proposed. The resulting formulation does not interpolate the director vectors but rather the rotation angles. No measures are taken against locking as computations with high order basis functions are the main objective of this contribution.

In Section 2 a short introduction to isogeometric analysis is given. All symbols and variables needed for the presented shell theory are defined. Section 3 contains the Reissner-Mindlin shell theory necessitating the definition of a director vector. The computation of discrete nodal director vectors needed to interpolate this director vector is investigated in Section 4. Here the new method entailing exactly interpolated director vectors is proposed. The isogeometric finite element implementation of the presented nonlinear shell theory is given in Section 5. A new approach for the interpolation of the variation of the director vector is presented. Numerical examples in Section 6 demonstrate the capability of the proposed shell formulation and compare it to existing formulations.

\section{Basic NURBS terminology for isogeometric shell analysis}

The presented shell formulation is based on a NURBS-based geometry description as well as interpolation is realized with the corresponding NURBS basis function. All variables and formulae needed for the shell formulation are briefly introduced. Details about the underlying algorithms can be found in [7, 8]. The terminology is in some points altered to be suitable to the shell formulation which follows [9].

A NURBS curve of order $p$ is uniquely described by a set of $n$-dimensional control points

$$
\mathbf{B}_{i}=\left[x_{i}, y_{i}, z_{i}, w_{i}\right]^{T}=\left[\mathbf{X}_{i}^{T}, w_{i}\right]^{T}
$$

with $i=1, \cdots, n$ and a knot vector $\boldsymbol{\Xi}=\left\{\xi_{1}, \xi_{2}, \cdots, \xi_{n+p+1}\right\}$. The knot vector must be non-decreasing and open, which means that the first and the last entry have to be repeated $p+1$ times. The B-Spline basis functions $N_{i}^{p}$ for a given knot vector $\boldsymbol{\Xi}$ are defined with the recursive Cox-de Boor formula beginning with order $p=0$ :

$$
\begin{aligned}
& p=0: \quad N_{i}^{0}(\xi)= \begin{cases}1 & \text { if } \xi_{i} \leq \xi \leq \xi_{i+1} \\
0 & \text { otherwise }\end{cases} \\
& p>0: \quad N_{i}^{p}(\xi)=\frac{\xi-\xi_{i}}{\xi_{i+p}-\xi_{i}} N_{i}^{p-1}(\xi)+\frac{\xi_{i+p+1}-\xi}{\xi_{i+p+1}-\xi_{i+1}} N_{i+1}^{p-1}(\xi)
\end{aligned}
$$


By tensor-product combination of two knot vectors $\boldsymbol{\Xi}^{1}=\left\{\xi_{1}^{1}, \xi_{2}^{1}, \cdots, \xi_{n+p+1}^{1}\right\}$ and $\boldsymbol{\Xi}^{2}=\left\{\xi_{1}^{2}, \xi_{2}^{2}, \cdots, \xi_{m+q+1}^{2}\right\}$ NURBS surface patches can be created. The control points

$$
\mathbf{B}_{i j}=\left[x_{i j}, y_{i j}, z_{i j}, w_{i j}\right]^{T}=\left[\mathbf{X}_{I}^{T}, w_{i j}\right]^{T}
$$

form a $n \times m$ control point net which is projected from a 4-dimensional space $\mathbb{R}^{4}$ to a surface embedded in a 3dimensional space $\mathbb{R}^{3}$ with the NURBS basis functions $N_{I}$. The index $I$ of the NURBS basis functions is a specified function of the indices $i$ and $j$ of the B-Splines basis functions. With

$$
W\left(\xi^{1}, \xi^{2}\right)=\sum_{i=1}^{n} \sum_{j=1}^{m} N_{i}^{p}\left(\xi^{1}\right) N_{j}^{q}\left(\xi^{2}\right) w_{i j}
$$

the NURBS surface basis functions are given as

$$
N_{I}\left(\xi^{1}, \xi^{2}\right)=\frac{N_{i}^{p}\left(\xi^{1}\right) N_{j}^{q}\left(\xi^{2}\right) w_{i j}}{W\left(\xi^{1}, \xi^{2}\right)}
$$

under consideration of the weights $w_{i j}$. In [7] it is shown, that for univariate B-Splines for every $\xi \in \Xi$ only $p+1$ basis functions are not equal to zero. Accordingly, $n_{e n}=(p+1)(q+1)$ specified basis functions have an impact on one arbitrary element. The total number of control points per patch computes to $n_{n p}=n \times m$ and the number of elements per patch is given by $n_{e l}=(n-p)(m-q)$. Finally a physical point $\mathbf{X}$ on the NURBS surface can be determined with

$$
\mathbf{X}\left(\xi^{1}, \xi^{2}\right)=\sum_{I=1}^{n_{e n}} N_{I}\left(\xi^{1}, \xi^{2}\right) \mathbf{X}_{I}
$$

The derivatives of bivariate NURBS basis functions with respect to the parametric coordinates $\xi^{\alpha}$ read

$$
\frac{\partial}{\partial \xi^{\alpha}} N_{I}=w_{i j} \frac{W \frac{\partial}{\partial \xi^{\alpha}}\left(N_{i}^{p} N_{j}^{q}\right)-\frac{\partial}{\partial \xi^{\alpha}}(W) N_{i}^{p} N_{j}^{q}}{W^{2}}
$$

with

$$
\frac{\partial}{\partial \xi} N_{i}^{p}=\frac{p}{\xi_{i+p}-\xi_{i}} N_{i}^{p-1}-\frac{p}{\xi_{i+p+1}-\xi_{i+1}} N_{i+1}^{p-1},
$$

where the dependence on $\left(\xi^{1}, \xi^{2}\right)$ is not displayed for the sake of clarity. For further details and an efficient algorithm see [7].

\section{Reissner-Mindlin shell theory}

The proposed shell element is formulated with a Reissner-Mindlin kinematic to incorporate transverse shear strains, which cannot be neglected in thick shell anaylsis. A rotational update formulation for the inextensible director vector field allows for large rotations. The derivation of the shell formulation from the continuum theory bases on the direct approach proposed by [6]. The variational formulation is pure displacement based. As locking almost vanishes with rising order $p$ of the NURBS basis functions no further measures are considered herein. Arbitrary nonlinear constitutive models can be combined with the proposed element formulation. As the focus of this contribution lies on the kinematic description details about the constitutive equations are omitted. All presented numerical examples are computed with a linear St. Venant-Kirchhoff-type material law.

\subsection{Kinematics}

The location of any physical point on the shell is given by

$$
\boldsymbol{\Phi}\left(\xi^{i}\right)=\mathbf{X}\left(\xi^{\alpha}\right)+\xi^{3} \mathbf{D}\left(\xi^{\alpha}\right)
$$




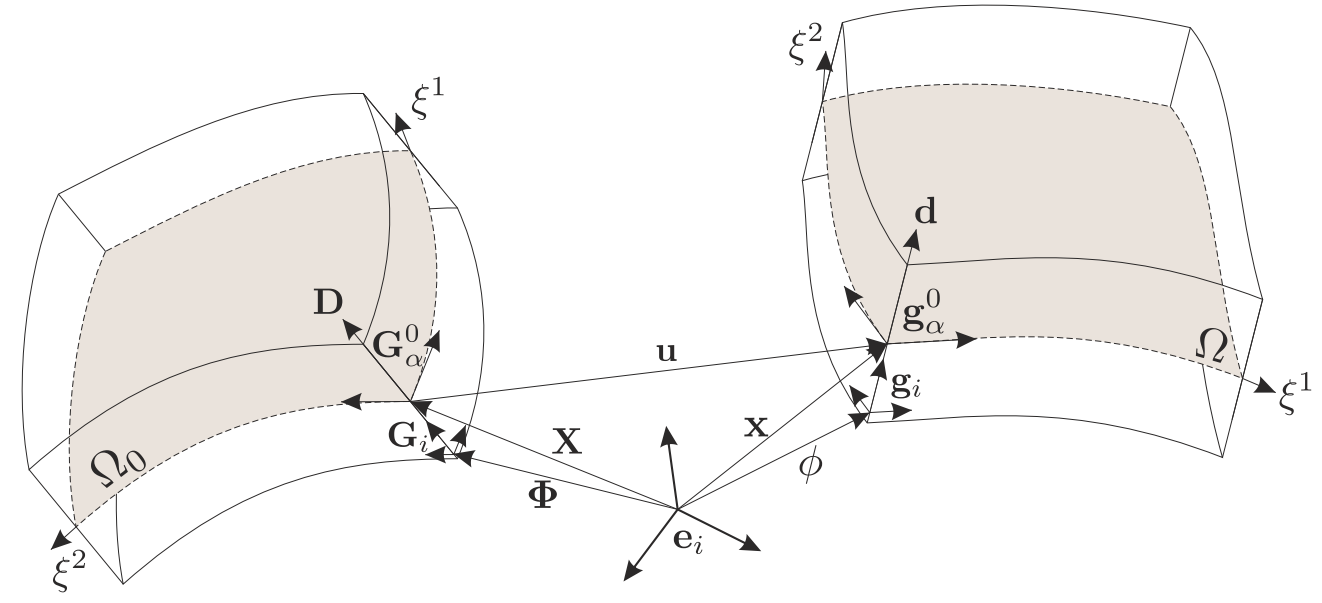

Figure 1: Reference and current configuration of the shell.

in the reference configuration $\mathcal{B}_{0}$ and by

$$
\boldsymbol{\phi}\left(\xi^{i}\right)=\mathbf{x}\left(\xi^{\alpha}\right)+\xi^{3} \mathbf{d}\left(\xi^{\alpha}\right)
$$

in the current configuration $\mathcal{B}$, see Fig. 1. If not specified differently Greek indices range from 1 to 2 and Latin indices from 1 to $3 . \xi^{3}$ defines the thickness coordinate and runs from $-h / 2$ to $h / 2$. $\mathbf{X}\left(\xi^{\alpha}\right)$ and $\mathbf{x}\left(\xi^{\alpha}\right)$ denote position vectors on the shell mid-surface $\Omega$. The reference director vector

$$
\left|\mathbf{D}\left(\xi^{\alpha}\right)\right|=1
$$

is perpendicular to the shell mid-surface and therefore identical to the normal vector. The current director $\mathbf{d}$ is computed via an orthogonal transformation $\mathbf{d}=\mathbf{R D}$ with an rotation tensor $\mathbf{R} \in S O$ (3). The use of an orthogonal transformation ensures the inextensibility of the director. The covariant basis vectors $\mathbf{G}_{\alpha}$ are defined as the derivatives of the position vectors $\boldsymbol{\Phi}$ and $\phi$ with respect to the coordinates $\xi^{\alpha}$. This results in

$$
\begin{aligned}
\mathbf{G}_{\alpha} & =\mathbf{X}_{, \alpha}+\xi^{3} \mathbf{D}_{, \alpha} & \mathbf{G}_{3} & =\mathbf{D} \\
\mathbf{g}_{\alpha} & =\mathbf{x}_{, \alpha}+\xi^{3} \mathbf{d}_{, \alpha} & \mathbf{g}_{3} & =\mathbf{d},
\end{aligned}
$$

where subscript comma denotes derivation with respect to the parametric coordinates $\xi^{\alpha}$. With the relation

$$
\mathbf{G}_{i} \cdot \mathbf{G}^{j}=\delta_{i}^{j}
$$

containing the Kornecker-Delta $\delta_{i}^{j}$ the contravariant basis vectors $\mathbf{G}^{i}$ are defined. The covariant basis vectors on the shell reference surface for $\xi^{3}=0$ are named $\mathbf{G}_{\alpha}^{0}$ and $\mathbf{g}_{\alpha}^{0}$. Their contravariant counterparts are analogously defined. With the determinant $\mu=\operatorname{det} \mathbf{Z}$ of the shifter tensor $\mathbf{Z}=\mathbf{G}_{k} \otimes \mathbf{G}^{0 k}$ area and volume integrals read

$$
\begin{aligned}
& \int \mathrm{d} A=\int\left|\mathbf{G}_{1}^{0} \times \mathbf{G}_{2}^{0}\right| \mathrm{d} \xi^{1} \mathrm{~d} \xi^{2} \\
& \int \mathrm{d} V=\int \mu\left|\mathbf{G}_{1}^{0} \times \mathbf{G}_{2}^{0}\right| \mathrm{d} \xi^{1} \mathrm{~d} \xi^{2} \mathrm{~d} \xi^{3} .
\end{aligned}
$$

\subsection{Strains and stresses}

With the deformation gradient $\mathbf{F}=\operatorname{Grad} \mathbf{x}$ the nonlinear Green-Lagrange strain tensor used as strain measure can be expressed by

$$
\mathbf{E}=\frac{1}{2}\left(\mathbf{F}^{T} \mathbf{F}-\mathbf{1}\right)=E_{i j} \mathbf{G}^{i} \otimes \mathbf{G}^{j}
$$


with covariant components

$$
E_{i j}=\frac{1}{2}\left(\boldsymbol{\phi}_{, i} \cdot \boldsymbol{\phi}_{, j}-\boldsymbol{\Phi}_{, i} \cdot \boldsymbol{\Phi}_{, j}\right)
$$

The tensorial components $E_{i j}$ are organized as resulting shell strains

$$
\begin{aligned}
\varepsilon_{\alpha \beta} & =\frac{1}{2}\left(\mathbf{x}_{, \alpha} \cdot \mathbf{x}_{, \beta}-\mathbf{X}_{, \alpha} \cdot \mathbf{X}_{, \beta}\right) \\
\kappa_{\alpha \beta} & =\frac{1}{2}\left(\mathbf{x}_{, \alpha} \cdot \mathbf{d}_{, \beta}+\mathbf{x}_{, \beta} \cdot \mathbf{d}_{, \alpha}-\mathbf{X}_{, \alpha} \cdot \mathbf{D}_{, \beta}-\mathbf{X}_{, \beta} \cdot \mathbf{D}_{, \alpha}\right) \\
\gamma_{\alpha} & =\mathbf{x}_{, \alpha} \cdot \mathbf{d}-\mathbf{X}_{, \alpha} \cdot \mathbf{D}
\end{aligned}
$$

to provide an efficient formulation. Here $\varepsilon_{\alpha \beta}$ denotes the membrane strains, $\kappa_{\alpha \beta}$ the curvatures and $\gamma_{\alpha}$ the shear strains. The relation to the Green-Lagrange strain components is given as

$$
\begin{aligned}
E_{\alpha \beta} & =\varepsilon_{\alpha \beta}+\xi^{3} \kappa_{\alpha \beta}+\left(\xi^{3}\right)^{2} \rho_{\alpha \beta} \\
2 E_{13} & =\gamma_{\alpha} \\
E_{33} & =0
\end{aligned}
$$

With respect to thin shells the second order curvatures $\rho_{\alpha \beta}$ are neglected. The vector of the resulting shell strains in Voigt notation reads

$$
\boldsymbol{\varepsilon}=\left[\varepsilon_{11}, \varepsilon_{22}, 2 \varepsilon_{12}, \kappa_{11}, \kappa_{22}, 2 \kappa_{12}, \gamma_{1}, \gamma_{2}\right]^{T}
$$

and is work-conjugate to the vector of the stress resultants of the Second Piola-Kirchhoff stress tensor

$$
\sigma=\left[n^{11}, n^{22}, n^{12}, m^{11}, m^{22}, m^{12}, q^{1}, q^{2}\right]^{T}
$$

The latter vector consists of membrane forces $n^{\alpha \beta}=\int_{\xi^{3}} S^{\alpha \beta} \mu \mathrm{d} \xi^{3}$, bending moments $m^{\alpha \beta}=\int_{\xi^{3}} S^{\alpha \beta} \mu \xi^{3} \mathrm{~d} \xi^{3}$ and shear forces $q^{\alpha}=\int_{\xi^{3}} S^{\alpha 3} \mu \mathrm{d} \xi^{3}$.

\subsection{Variational formulation}

The presented shell is based on a pure displacement formulation. Loads are restricted to conservative surface loads $\overline{\mathbf{p}}$ on the reference surface $\Omega_{0}$ and Neumann conditions $\overline{\mathbf{t}}$ on the boundary $\Gamma_{t}$. Loading is assumed to be static. The potential hence reads

$$
\Pi(\mathbf{v})=\int_{\mathcal{B}_{0}} W(\boldsymbol{\varepsilon}) \mathrm{d} V-\int_{\Omega_{0}} \mathbf{u}^{T} \overline{\mathbf{p}} \mathrm{d} A-\int_{\Gamma_{t}} \mathbf{u}^{T} \overline{\mathbf{t}} \mathrm{d} s
$$

where the strain energy function $W$ is defined by the used material law. The independent displacement vector $\mathbf{v}=$ $[\mathbf{u}, \omega]^{T}$ contains the displacements $\mathbf{u}$ and the rotational parameters $\omega$ of the shell mid-surface. With $\delta W=\boldsymbol{\sigma} \cdot \delta \boldsymbol{\varepsilon}$ the variation of the potential

$$
\delta \Pi(\mathbf{v}, \delta \mathbf{v})=\int_{\mathcal{B}_{0}} \delta \boldsymbol{\varepsilon}^{T} \sigma \mathrm{d} V-\int_{\Omega_{0}} \delta \mathbf{u}^{T} \overline{\mathbf{p}} \mathrm{d} A-\int_{\Gamma_{t}} \delta \mathbf{u}^{T} \overline{\mathbf{t}} \mathrm{d} s=0
$$

leads to the weak form $G(\mathbf{v}, \delta \mathbf{v})$ if virtual deformations are used as test functions. The vector $\delta \boldsymbol{\varepsilon}=\left[\delta \varepsilon_{11}, \delta \varepsilon_{22}, 2 \delta \varepsilon_{12}, \delta \kappa_{11}, \delta \kappa_{22}, 2 \delta \kappa_{12}, \delta \gamma_{1}, \delta \gamma_{2}\right]^{T}$ contains the virtual shell strains

$$
\begin{aligned}
\delta \varepsilon_{\alpha \beta} & =\frac{1}{2}\left(\delta \mathbf{x}_{, \alpha} \cdot \mathbf{x}_{, \beta}+\delta \mathbf{x}_{, \beta} \cdot \mathbf{x}_{, \alpha}\right) \\
\delta \kappa_{\alpha \beta} & =\frac{1}{2}\left(\delta \mathbf{x}_{, \alpha} \cdot \mathbf{d}_{, \beta}+\delta \mathbf{x}_{, \beta} \cdot \mathbf{d}_{, \alpha}+\delta \mathbf{d}_{, \alpha} \cdot \mathbf{x}_{, \beta}+\delta \mathbf{d}_{, \beta} \cdot \mathbf{x}_{, \alpha}\right) \\
\delta \gamma_{\alpha} & =\delta \mathbf{x}_{, \alpha} \cdot \mathbf{d}+\delta \mathbf{d} \cdot \mathbf{x}_{, \alpha} .
\end{aligned}
$$




\subsection{Second variation ot the potential}

The nonlinear problem (22) is solved by applying the Newton-Raphson scheme. The necessary linearization of the weak form

$$
\mathrm{L}[G(\mathbf{v}, \delta \mathbf{v})]=G+\mathrm{D} G \cdot \Delta \mathbf{v}
$$

entails the need for the second variation of the potential (21). This is derived with the Gateaux derivative and yields

$$
\mathrm{D} G(\mathbf{v}, \delta \mathbf{v}) \cdot \Delta \mathbf{v}=\int_{\mathcal{B}_{0}} \delta \boldsymbol{\varepsilon}^{T} \mathbb{C} \Delta \boldsymbol{\varepsilon}+\Delta \delta \boldsymbol{\varepsilon}^{T} \boldsymbol{\sigma} \mathrm{d} V,
$$

where

$$
\mathbb{C}=\frac{\partial^{2} W}{\partial \boldsymbol{\varepsilon} \partial \boldsymbol{\varepsilon}}=\frac{\partial \sigma}{\partial \boldsymbol{\varepsilon}}
$$

is given by the material law. The linearized virtual shell strain vector

$$
\Delta \delta \boldsymbol{\varepsilon}=\left[\Delta \delta \varepsilon_{11}, \Delta \delta \varepsilon_{22}, 2 \Delta \delta \varepsilon_{12}, \Delta \delta \kappa_{11}, \Delta \delta \kappa_{22}, 2 \Delta \delta \kappa_{12}, \Delta \delta \gamma_{1}, \Delta \delta \gamma_{2}\right]^{T}
$$

has the entries

$$
\begin{aligned}
\Delta \delta \varepsilon_{\alpha \beta}= & \frac{1}{2}\left(\delta \mathbf{x}_{, \alpha} \cdot \Delta \mathbf{x}_{\beta}+\delta \mathbf{x}_{, \beta} \cdot \Delta \mathbf{x}_{, \alpha}\right) \\
\Delta \delta \kappa_{\alpha \beta}= & \frac{1}{2}\left(\delta \mathbf{x}_{, \alpha} \cdot \Delta \mathbf{d}_{\beta}+\delta \mathbf{x}_{, \beta} \cdot \Delta \mathbf{d}_{, \alpha}+\delta \mathbf{d}_{, \alpha} \cdot \Delta \mathbf{x}_{, \beta}+\delta \mathbf{d}_{\beta} \cdot \Delta \mathbf{x}_{, \alpha}\right. \\
& \left.\quad+\mathbf{x}_{, \alpha} \cdot \Delta \delta \mathbf{d}_{, \beta}+\mathbf{x}_{, \beta} \cdot \Delta \delta \mathbf{d}_{, \alpha}\right) \\
\Delta \delta \gamma_{\alpha}= & \delta \mathbf{x}_{, \alpha} \cdot \Delta \mathbf{d}+\delta \mathbf{d} \cdot \Delta \mathbf{x}_{, \alpha}+\mathbf{x}_{, \alpha} \cdot \Delta \delta \mathbf{d}
\end{aligned}
$$

and the linearized shell strains $\Delta \boldsymbol{\varepsilon}$ are obtained by replacing the operator $\delta$ by $\Delta$ in (23).

\section{Definition of nodal basis systems}

There are several ways to interpolate the current director vector and its variations in Reissner-Mindlin shell formulations. The chosen interpolation strategy requires a nodal basis system $\mathbf{A}_{I}$ in every control point. The two basis vectors $\mathbf{A}_{1 I}$ and $\mathbf{A}_{2 I}$ define the rotation axis for the rotational degrees-of-freedom and $\mathbf{A}_{3 I}$ is used for the interpolation of the director vector. The nodal basis systems have to be computed in a preprocess or given as an input. As in isogeometric analysis the control point net is disjunct from the physical mesh several choices are possible. In the following two methods to compute the nodal basis systems are discussed. The first is to use an orthonormal basis system in the closest point projection of the relevant control point. This is an obvious choice and is used in [4] for an isogeometric Reissner-Mindlin shell based on the degenerated solid approach. In the numerical examples in Section 6 the standard approach uses this method. The second scheme is called calculation of exact basis systems and yields exact orthonormal interpolated basis systems with correct orientation throughout the whole domain for every discretization. Together with the presented interpolation of the variation of the director the latter method constitutes the main scope of this paper.

\subsection{Basis systems obtained by closest point projection}

The first step of this method is to find the point on the NURBS surface with the smallest distance to the control point under consideration. In general a solution in closed form to this is not possible. Therefore a Newton-Raphson iteration with additional computational cost is needed. The theory and an efficient algorithm for the point projection is given in [7]. In the second step the director vector

$$
\mathbf{D}_{I}=\mathbf{A}_{3 I}=\frac{\mathbf{X}_{, \xi^{1}} \times \mathbf{X}_{, \xi^{2}}}{\left\|\mathbf{X}_{, \xi^{1}} \times \mathbf{X}_{, \xi^{2}}\right\|}
$$


is computed from the derivatives $\mathbf{X}_{, \xi^{1}}$ and $\mathbf{X}_{, \xi^{2}}$ in the projected point. The other two nodal basis vectors $\mathbf{A}_{1 I}$ and $\mathbf{A}_{2 I}$ are chosen in the plane of the derivatives in a way, that proper enforcement of the boundary conditions is possible and the nodal basis system is orthogonal.

The accuracy of the interpolated director vector

$$
\mathbf{D}^{h}=\sum_{I=1}^{n_{e n}} N_{I} \mathbf{D}_{I}
$$

is examined for the Scordelis-Lo roof from the shell obstacle course proposed in [10]. Considering symmetry only a quarter of the system is analyzed, see Fig. 2. Details can be found in Section 6.1 or [10]. The error of length and



Figure 2: Deviation of the interpolated director vector from the normal vector.

direction of the interpolated director is measured with the error norms

$$
\begin{aligned}
L_{\text {length }}^{2} & =\sqrt{\int_{\Omega}\left(\left\|\mathbf{D}^{h}\right\|-1\right)^{2} \mathrm{~d} \Omega} \\
L_{\text {angle }}^{2} & =\sqrt{\int_{\Omega}\left(\arccos \frac{\mathbf{D}^{h} \cdot \mathbf{N}}{\left\|\mathbf{D}^{h}\right\|\|\mathbf{N}\|}\right)^{2} \mathrm{~d} \Omega},
\end{aligned}
$$

where $\mathbf{N}$ is the exact normal vector with respect to the shell mid-surface. Fig. 2 clearly shows a deterioration of the interpolated director in the integration points for $k$-refinement with fixed mesh size. This is due to the fact, that the number of control points having influence on one element $n_{e n}=(n-p)(m-q)$ as well as their distance to the element grows with the polynomial order $p$ respective $q$. Thus for curved domains the angle between the individual nodal directors $\mathbf{D}_{I}$ grows. This leads to an addition of vectors with distinct differing directions, which clearly deteriorates the interpolation. The effect on the convergence behavior is investigated in Section 6.1.

\subsection{Calculation of exact basis systems}

A new method to determine the nodal basis systems is introduced in the following. The idea is to chose each nodal basis system in a way, that the interpolated basis system is orthonormal in every integration point, and the director vector as well as the local rotation axis are in the correct direction. To attain this in every patch a system of equations is established and solved independently. The three basis vectors $\mathbf{A}_{i}$ entail nine equations in every integration point of the relevant patch. This yields $\left(n_{g p} \times n_{e l}\right)$ equations of the type

$$
A_{i j}^{G P}=\sum_{I=1}^{n_{n p}} N_{I} A_{i j I}^{C P},
$$


where $n_{g p}$ is the number of integration points per element and $n_{e l}$ is the number of elements in the relevant patch. The upper bound $n_{n p}$ of the summation in (32) is the number of all control points in the patch under consideration. $A_{i j}^{G P}$ is the $j$-th component of the $i$-th basis vector of the integration point basis system and analogously $A_{i j I}^{C P}$ are the unknown values for the nodal point basis systems at the control point $I$. For every combination of $i$ and $j$ all equations (32) are assembled to a system of equations

$$
\mathbf{A}_{i j}^{G P}=\mathbf{N A}_{i j}^{C P},
$$

where the matrix $\mathbf{N}$ has $\left(n_{g p} \times n_{e l}\right)$ rows and $n_{n p}$ columns containing the basis functions $N_{I}$. The matrix $\mathbf{N}$ is identical for all nine directions and thus only one matrix inversion is necessary to compute all components of the nodal basis systems. The number of integration points per element $n_{g p}$ must be chosen high enough to entail a determined or overdetermined system of equations. This is always the case if $n_{g p}$ is chosen sufficiently high for the structural analysis to be stable. The columns of the matrix $\mathbf{N}$ contain the linear independent basis functions $N_{I}$ and therefore all columns are linear independent, which implies that

$$
\operatorname{rank} \mathbf{N}=n_{n p}
$$

holds and a unique solution exists, see [11]. Thus the solution of (33) can always be determined by solving the normal equation

$$
\mathbf{N}^{T} \mathbf{A}_{i j}^{G P}=\left(\mathbf{N}^{T} \mathbf{N}\right) \mathbf{A}_{i j}^{C P},
$$

which is determined and thus solvable with standard solvers. The resulting nodal basis systems yield exactly interpolated director vectors and local rotation axes throughout the whole domain. The number of unknowns in equation (35) is approximately five times smaller than that of the finite element computation. This signifies that the cost for the computation of the nodal basis system is negligible. As the influence of all basis functions $N_{I}$ on an element is fixed by the NURBS geometry description, standard sparse matrix techniques can be applied. The required matrices can be assembled element-wise by

$$
\left(\mathbf{N}^{T} \mathbf{N}\right)(k, l)=\sum_{e q=1}^{n_{g p} \times n_{e l}}\left(N_{k} N_{l}\right)_{e q}
$$

and

$$
\left(\mathbf{N}^{T} \mathbf{A}_{i j}^{G P}\right)(k)=\sum_{e q=1}^{n_{g p} \times n_{e l}}\left(A_{i j}^{G P} N_{k}\right)_{e q}
$$

to avoid multiplications of the terms $\mathbf{N}^{T} \mathbf{N}$ and $\mathbf{N}^{T} \mathbf{A}_{i j}^{G P}$ on the global level.

\section{NURBS-based isogeometric finite element implementation}

The isogeometric finite element implementation of the Reissner-Mindlin shell with exactly calculated basis systems fields is described in the following. For completeness the outline of an isogeometric implementation of a Reissner-Mindlin shell with standard interpolation of the director vector and nodal basis systems attained by closest point projection is additionally provided. This formulation follows [12] and constitutes the standard approach used in the numerical examples in Section 6.

\subsection{Interpolation of the reference surface and the director vector}

The shell middle surface and the initial director vector are interpolated by

$$
\mathbf{X}=\sum_{I=1}^{n_{e n}} N_{I} \mathbf{X}_{I} \quad \mathbf{x}^{h}=\sum_{I=1}^{n_{e n}} N_{I} \mathbf{x}_{I} \quad \mathbf{D}=\sum_{I=1}^{n_{e n}} N_{I} \mathbf{D}_{I}
$$

with the NURBS basis functions $N_{I}$ defined in (5). The superscript $h$ identifies approximated quantities. The position vectors $\mathbf{X}_{I}$ are given as input and the director vectors $\mathbf{D}_{I}=\mathbf{A}_{3 I}$ are taken from the preprocess described in Section 4. The interpolation of the initial director vector $\mathbf{D}$ is only exact if the nodal basis systems are computed with the method described in Section 4.2. 
The derivatives of the NURBS basis functions given in (7) are with respect to the parametric coordinates $\xi^{\alpha}$ and in general not orthogonal. For the use in the finite element formulation they need to be transformed to the local cartesian basis $\mathbf{t}$. From the two derivatives

$$
\mathbf{X}_{, \xi^{1}}=\sum_{I=1}^{n_{e n}} N_{I, \xi^{1}} \mathbf{X}_{I} \quad \mathbf{X}_{, \xi^{2}}=\sum_{I=1}^{n_{e n}} N_{I, \xi^{2}} \mathbf{X}_{I}
$$

a local lamina basis system spanned by the vectors $\mathbf{t}_{1}, \mathbf{t}_{2}$ and $\mathbf{t}_{3}$ is computed as described in [13]. With the Jacobian

$$
\mathbf{J}=\left[\begin{array}{ll}
\mathbf{X}_{, \xi^{1}} \cdot \mathbf{t}_{1} & \mathbf{X}_{, \xi^{1}} \cdot \mathbf{t}_{2} \\
\mathbf{X}_{, \xi^{2}} \cdot \mathbf{t}_{1} & \mathbf{X}_{, \xi^{2}} \cdot \mathbf{t}_{2}
\end{array}\right]
$$

the local derivatives

$$
\left[\begin{array}{c}
N_{I, 1} \\
N_{I, 2}
\end{array}\right]=\mathbf{J}^{-1}\left[\begin{array}{c}
N_{I, \xi^{1}} \\
N_{I, \xi^{2}}
\end{array}\right]
$$

can be computed. With (41) the derivatives of (38) are interpolated by:

$$
\mathbf{X}_{, \alpha}=\sum_{I=1}^{n_{e n}} N_{I, \alpha} \mathbf{X}_{I} \quad \mathbf{x}_{, \alpha}^{h}=\sum_{I=1}^{n_{e n}} N_{I, \alpha} \mathbf{x}_{I} \quad \mathbf{D}_{, \alpha}=\sum_{I=1}^{n_{e n}} N_{I, \alpha} \mathbf{D}_{I}
$$

\subsubsection{Standard interpolation of the current director vector}

In a standard Reissner-Mindlin shell element, see e.g. [12], the orthogonality of the nodal basis system A holds only at the nodes. Therefore the Rodrigues' formula

$$
\begin{aligned}
& \mathbf{R}=\mathbf{1}+\frac{\sin \omega}{\omega} \boldsymbol{\Omega}+\frac{1-\cos \omega}{\omega^{2}} \boldsymbol{\Omega}^{2} \\
& \boldsymbol{\Omega}=\operatorname{skew} \boldsymbol{\omega}=\left[\begin{array}{ccc}
0 & -\omega_{3} & \omega_{2} \\
\omega_{3} & 0 & -\omega_{1} \\
-\omega_{2} & \omega_{1} & 0
\end{array}\right]
\end{aligned}
$$

with $\omega=\|\omega\|$ is applied to the discrete nodal basis systems $\mathbf{A}_{I}$. For brevity the subscript for the nodal index $I$ is omitted, but if equation (43) is used for the standard interpolation nodal values $\omega_{I}$ have to be used. For values of $\omega>2 \pi$ a multiplicative update of the rotation tensor is necessary. The interpolation of the current director and the derivatives is as follows:

$$
\mathbf{d}^{h}=\sum_{I=1}^{n_{e n}} N_{I} \mathbf{d}_{I} \quad \mathbf{d}_{, \alpha}^{h}=\sum_{I=1}^{n_{e n}} N_{I, \alpha} \mathbf{d}_{I} \quad \mathbf{d}_{I}=\mathbf{R} \mathbf{A}_{3 I}
$$

The interpolation does not maintain the length and the orthogonality of the basis systems. The entailed error turns out to be negligible for standard finite elements and isogeometric finite elements with a polynomial order $p \leq 2$. But for rising orders $p$ the accuracy of isogeometric computations deteriorates.

\subsubsection{Consistent interpolation of the current director vector}

Due to the exact interpolation the orthogonality of the basis system A prevails throughout the whole domain. This allows a consistent determination of the current director

$$
\mathbf{d}^{h}=\mathbf{R} \sum_{I=1}^{n_{e n}} N_{I} \mathbf{D}_{I} \quad \mathbf{d}_{, \alpha}^{h}=\mathbf{R}_{, \alpha} \sum_{I=1}^{n_{e n}} N_{I} \mathbf{D}_{I}+\mathbf{R} \sum_{I=1}^{n_{e n}} N_{I, \alpha} \mathbf{D}_{I}
$$

with the rotation tensor $\mathbf{R}$ given in (43) and $\mathbf{R}_{, \alpha}$ computed as follows:

$$
\begin{array}{rlrl}
\mathbf{R}_{, \alpha} & =c_{1} \boldsymbol{\Omega}+c_{2} \boldsymbol{\Omega}_{, \alpha}+c_{3} \boldsymbol{\Omega}^{2}+c_{4}\left(\mathbf{\Omega}_{, \alpha} \mathbf{\Omega}+\mathbf{\Omega}_{, \alpha}\right) \\
\mathbf{\Omega}_{, \alpha} & =\operatorname{skew} \omega_{, \alpha} & \omega_{, \alpha} & =\frac{\omega_{, \alpha} \cdot \omega}{\omega} \\
c_{1} & =\omega_{, \alpha} \frac{\omega \cos \omega-\sin \omega}{\omega^{2}} & c_{2} & =\frac{\sin \omega}{\omega} \\
c_{3} & =\omega_{, \alpha} \frac{\omega \sin \omega-2+2 \cos \omega}{\omega^{3}} & c_{4} & =\frac{1-\cos \omega}{\omega^{2}}
\end{array}
$$


Here for $\omega$ the interpolated value in the relevant integration point has to be inserted. For values $\omega \rightarrow 0$ the factors $c_{1}=0, c_{2}=1, c_{3}=0$ and $c_{4}=1 / 2$ have to be used. The inextensibility of the director field

$$
\left\|\mathbf{d}^{h}\right\|=1
$$

is exactly fulfilled.

\subsection{Interpolation of strains and variated strains}

The interpolation of the Green-Lagrange strains $\varepsilon$ given in equation (19) is simply performed by replacing all variables through their interpolated counterparts defined in equations (38), (42) and (44) or (45), respectively. The procedure to attain the virtual Green-Lagrange strains $\delta \varepsilon^{h}$ from $\delta \boldsymbol{\varepsilon}$ is akin. It results in

$$
\delta \boldsymbol{\varepsilon}^{h}=\left[\begin{array}{c}
\delta \varepsilon_{11}^{h} \\
\delta \varepsilon_{22}^{h} \\
2 \delta \varepsilon_{12}^{h} \\
\delta \kappa_{11}^{h} \\
\delta \kappa_{22}^{h} \\
2 \delta \kappa_{12}^{h} \\
\delta \gamma_{1}^{h} \\
\delta \gamma_{2}^{h}
\end{array}\right]=\left[\begin{array}{c}
\delta \mathbf{x}_{, 1}^{h} \cdot \mathbf{x}_{, 1}^{h} \\
\delta \mathbf{x}_{, 2}^{h} \cdot \mathbf{x}_{, 2}^{h} \\
\delta \mathbf{x}_{11}^{h} \cdot \mathbf{x}_{, 2}^{h}+\delta \mathbf{x}_{, 2}^{h} \cdot \mathbf{x}_{, 1}^{h} \\
\delta \mathbf{x}_{1}^{h} \cdot \mathbf{d}_{, 1}^{h}+\delta \mathbf{d}_{11}^{h} \cdot \mathbf{x}_{, 1}^{h} \\
\delta \mathbf{x}_{2}^{h} \cdot \mathbf{d}_{2}^{h}+\delta \mathbf{d}_{2}^{h} \cdot \mathbf{x}_{, 2}^{h} \\
\delta \mathbf{x}_{, 1}^{h} \cdot \mathbf{d}_{, 2}^{h}+\delta \mathbf{x}_{2,}^{h} \cdot \mathbf{d}_{, 1}^{h}+\delta \mathbf{d}_{, 1}^{h} \cdot \mathbf{x}_{, 2}^{h}+\delta \mathbf{d}_{, 2}^{h} \cdot \mathbf{x}_{, 1}^{h} \\
\delta \mathbf{x}_{, 1}^{h} \cdot \mathbf{d}^{h}+\delta \mathbf{d}^{h} \cdot \mathbf{x}_{, 1}^{h} \\
\delta \mathbf{x}_{, 2}^{h} \cdot \mathbf{d}^{h}+\delta \mathbf{d}^{h} \cdot \mathbf{x}_{, 2}^{h}
\end{array}\right]
$$

with

$$
\delta \mathbf{x}^{h}=\sum_{I=1}^{n_{e n}} N_{I} \delta \mathbf{u}_{I} \quad \delta \mathbf{x}_{, \alpha}^{h}=\sum_{I=1}^{n_{e n}} N_{I, \alpha} \delta \mathbf{u}_{I} .
$$

The interpolation of $\delta \mathbf{d}_{, \alpha}^{h}$ and $\delta \mathbf{d}^{h}$ is given below for the two presented interpolations.

\subsubsection{Standard interpolation of the variated current director vector}

The standard approach following [12] interpolates the variated director

$$
\delta \mathbf{d}^{h}=\sum_{I=1}^{n_{e n}} N_{I} \delta \mathbf{d}_{I} \quad \delta \mathbf{d}_{, \alpha}^{h}=\sum_{I=1}^{n_{e n}} N_{I, \alpha} \delta \mathbf{d}_{I}
$$

with

$$
\delta \mathbf{d}_{I}=\delta \mathbf{w}_{I} \times \mathbf{d}_{I}=\mathbf{W}^{T} \delta \mathbf{w}_{I}=\mathbf{W}^{T} \mathbf{H} \delta \omega_{I}=\mathbf{W}^{T} \mathbf{H} \mathbf{T}_{3} \delta \boldsymbol{\beta}_{I}=\mathbf{T}_{I} \delta \boldsymbol{\beta}_{I}
$$

where

$$
\mathbf{W}=\text { skew } \mathbf{d} \text {. }
$$

The matrix

$$
\mathbf{H}=\mathbf{1}+\frac{1-\cos \omega}{\omega^{2}} \boldsymbol{\Omega}+\frac{\omega-\sin \omega}{\omega^{3}} \mathbf{\Omega}^{2}
$$

is derived in [14]. With

$$
\mathbf{T}_{3}=\left[\begin{array}{ll}
\mathbf{a}_{1} & \mathbf{a}_{2}
\end{array}\right]
$$

the transformation from local rotations $\beta_{I}$ to the rotational parameters $\omega_{I}$ is achieved. Note that again for $\omega, \mathbf{d}$ and $\mathbf{a}_{\alpha}$ nodal values $\omega_{I}, \mathbf{d}_{I}$ and

$$
\mathbf{a}_{\alpha I}=\mathbf{R} \mathbf{A}_{\alpha I}
$$

have to be inserted and therefore all matrices in equation (51) have to be calculated for every node. With (50) at hand the relation between virtual deformations and virtual strains can be established. This allows the computation of the stiffness matrix, which requires the second variation of the potential. Its derivation follows the lines of [12]. In the following only the proposed new approach is described. 


\subsubsection{Proposed interpolation of the variated current director vector}

The proposed approach for the interpolation of $\delta \mathbf{d}$ does not interpolate the nodal variation of the director, but the local rotations $\delta \boldsymbol{\beta}$. This allows a transformation of the rotations from integration point to control point which results in higher accuracy for curved domains.

Remark 1. In [15] a similar procedure, referred to as full SO(3) update, is discussed. There the current director vector is computed in the same way as proposed here, but deviating from our approach the rotational parameter vector $\delta \omega$ is interpolated. The proposed approach is more suitable for isogeometric shell analysis, as account is taken for the differing orientation of the nodal rotations.

For an orthogonal tensor $\mathbf{R} \in S O$ (3) the variation of the director can be rewritten as

$$
\begin{aligned}
\delta \mathbf{d} & =\delta \mathbf{R D} \\
& =\delta \mathbf{R R}^{T} \mathbf{d} \\
& =\delta \mathbf{w} \times \mathbf{d} \\
& =\mathbf{W}^{T} \delta \mathbf{w},
\end{aligned}
$$

where $\mathbf{W}=$ skew $\mathbf{d}$. With the two equations

$$
\delta \mathbf{w}=\mathbf{H} \delta \omega \quad \delta \omega=\mathbf{T}_{3} \delta \boldsymbol{\beta}
$$

the relation

$$
\delta \mathbf{d}=\mathbf{W}^{T} \mathbf{H} \mathbf{T}_{3} \delta \boldsymbol{\beta}=\mathbf{T} \delta \boldsymbol{\beta}
$$

is established by using the matrices given in (52), (53) and (54). Here the interpolated values $\omega, \mathbf{d}$ and

$$
\mathbf{a}_{\alpha}=\mathbf{R} \mathbf{A}_{\alpha} \quad \mathbf{A}_{\alpha}=\sum_{I=1}^{n_{e n}} N_{I} \mathbf{A}_{\alpha I}
$$

have to be inserted. For the interpolation of $\delta \boldsymbol{\beta}$ a transformation from local rotations in the control points $\delta \boldsymbol{\beta}_{I}$ to local rotations in the integration points is introduced. From

$$
\underbrace{\delta \beta_{i} \mathbf{a}_{i}}_{\text {integration point }}=\underbrace{\sum_{I=1}^{n_{e n}} N_{I} \beta_{i I} \mathbf{a}_{i I}}_{\text {control point }}
$$

the relation

$$
\delta\left[\begin{array}{l}
\beta_{1} \\
\beta_{2} \\
\beta_{3}
\end{array}\right]=\sum_{I=1}^{n_{e n}} N_{I}\left[\begin{array}{lll}
\mathbf{a}_{1} \cdot \mathbf{a}_{1 I} & \mathbf{a}_{1} \cdot \mathbf{a}_{2 I} & \mathbf{a}_{1} \cdot \mathbf{a}_{3 I} \\
\mathbf{a}_{2} \cdot \mathbf{a}_{1 I} & \mathbf{a}_{2} \cdot \mathbf{a}_{2 I} & \mathbf{a}_{2} \cdot \mathbf{a}_{3 I} \\
\mathbf{a}_{3} \cdot \mathbf{a}_{1 I} & \mathbf{a}_{3} \cdot \mathbf{a}_{2 I} & \mathbf{a}_{3} \cdot \mathbf{a}_{3 I}
\end{array}\right] \delta\left[\begin{array}{c}
\beta_{1 I} \\
\beta_{2 I} \\
\beta_{3 I}
\end{array}\right]
$$

can be derived. The drilling rotation $\delta \beta_{3}$ is fixed as the shell has no drilling stiffness. Therefore with $\delta \beta_{3}=0$ a statical condensation is possible and $\delta \beta_{3 I}$ can be eliminated from equation (61). As result the interpolation

$$
\delta \boldsymbol{\beta}^{h}=\sum_{I=1}^{n_{e n}} N_{I} \mathbf{M}_{I} \delta \boldsymbol{\beta}_{I}
$$

with

$$
\mathbf{M}_{I}=\left[\begin{array}{ll}
M_{11 I} & M_{12 I} \\
M_{21 I} & M_{22 I}
\end{array}\right] \quad M_{\alpha \beta I}=\mathbf{a}_{\alpha} \cdot \mathbf{a}_{\beta I}-\frac{\left(\mathbf{a}_{3} \cdot \mathbf{a}_{\beta I}\right)\left(\mathbf{a}_{\alpha} \cdot \mathbf{a}_{3 I}\right)}{\mathbf{a}_{3} \cdot \mathbf{a}_{3 I}}
$$

is established. 
Remark 2. If the interpolated basis vector $\mathbf{a}_{3}$ and a nodal basis vector $\mathbf{a}_{3 I}$ are perpendicular the statical condensation of equation (61) is not possible. In these cases six degrees of freedom like in shell analysis with kinks are needed for the affected control point. These situations only occur for very coarse meshes and can be avoided by an appropriate discretization. Sloppyly it is possible to omit the transformation matrix $\mathbf{M}_{I}$ which results in a loss of accuracy.

Remark 3. When three nodal rotations for control points on kinks are used and the matrix $\mathbf{M}_{I}$ is chosen appropriately minor additions to the presented theory allow the computation of complicated structures with intersecting surfaces.

The combinination of equation (62) and (58) yields the interpolation

$$
\delta \mathbf{d}^{h}=\sum_{I=1}^{n_{e n}} N_{I} \mathbf{T}_{M_{I}} \delta \boldsymbol{\beta}_{I} \quad \mathbf{T}_{M_{I}}=\mathbf{T} \mathbf{M}_{I}
$$

of the variation of the director, which contains several spatial variable factors. Thus, the derivation $\delta \mathbf{d}_{, \alpha}^{h}$ entails derivation of several matrices. It reads

$$
\delta \mathbf{d}_{, \alpha}^{h}=\mathbf{T}_{, \alpha} \sum_{I=1}^{n_{e n}} N_{I} \mathbf{M}_{I} \delta \boldsymbol{\beta}_{I}+\mathbf{T} \sum_{I=1}^{n_{e n}}\left(N_{I, \alpha} \mathbf{M}_{I}+N_{I} \mathbf{M}_{I, \alpha}\right) \delta \boldsymbol{\beta}_{I}
$$

with

$$
\mathbf{T}_{, \alpha}=\mathbf{W}_{, \alpha}^{T} \mathbf{H} \mathbf{T}_{3}+\mathbf{W}^{T} \mathbf{H}_{, \alpha} \mathbf{T}_{3}+\mathbf{W}^{T} \mathbf{H} \mathbf{T}_{3, \alpha} .
$$

The derivations of the involved matrices are given by

$$
\mathbf{W}_{, \alpha}=\operatorname{skew} \mathbf{d}_{, \alpha} \quad \mathbf{T}_{3, \alpha}=\left[\begin{array}{ll}
\mathbf{a}_{1, \alpha} & \mathbf{a}_{2, \alpha}
\end{array}\right]
$$

and

$$
\begin{array}{rlrl}
\mathbf{H}_{, \alpha} & =h_{1} \boldsymbol{\Omega}+h_{2} \boldsymbol{\Omega}_{, \alpha}+h_{3} \boldsymbol{\Omega}^{2}+h_{4}\left(\boldsymbol{\Omega}_{, \alpha} \boldsymbol{\Omega}+\mathbf{\Omega}_{\boldsymbol{\Omega}_{, \alpha}}\right) \\
h_{1} & =\omega_{, \alpha} \frac{\omega \sin \omega-2(1-\cos \omega)}{\omega^{3}} \quad h_{2}=\frac{1-\cos \omega}{\omega^{2}} \\
h_{3} & =\omega_{, \alpha} \frac{3 \sin \omega-\omega \cos \omega-2 \omega}{\omega^{4}} & h_{4} & =\frac{\omega-\sin \omega}{\omega^{3}},
\end{array}
$$

where for small values of $\omega$ the limit values $h_{1}=0, h_{2}=1 / 2, h_{3}=0$ and $h_{4}=1 / 6$ have to be used. The derivation of the transformation matrix reads:

$$
\begin{aligned}
\mathbf{M}_{I, \alpha}= & {\left[\begin{array}{ll}
M_{11 I, \alpha} & M_{12 I, \alpha} \\
M_{21 I, \alpha} & M_{22 I, \alpha}
\end{array}\right] } \\
M_{\gamma \delta I, \alpha}=\mathbf{a}_{\gamma, \alpha} \cdot \mathbf{a}_{\delta I} & -\frac{\left[\left(\mathbf{a}_{3, \alpha} \cdot \mathbf{a}_{\delta I}\right)\left(\mathbf{a}_{\gamma} \cdot \mathbf{a}_{3 I}\right)+\left(\mathbf{a}_{3} \cdot \mathbf{a}_{\delta I}\right)\left(\mathbf{a}_{\gamma, \alpha} \cdot \mathbf{a}_{3 I}\right)\right]}{\left(\mathbf{a}_{3} \cdot \mathbf{a}_{3 I}\right)} \\
& -\frac{\left(\mathbf{a}_{3, \alpha} \cdot \mathbf{a}_{3 I}\right)\left(\mathbf{a}_{3} \cdot \mathbf{a}_{\delta I}\right)\left(\mathbf{a}_{\gamma} \cdot \mathbf{a}_{3 I}\right)}{\left(\mathbf{a}_{3} \cdot \mathbf{a}_{3 I}\right)^{2}}
\end{aligned}
$$

The relation between virtual strains and the variations of deformations and rotations, abbreviated with the $\mathbf{B}_{I}$ matrix, can be written as

$$
\begin{aligned}
\delta \boldsymbol{\varepsilon}^{h}= & {\left[\begin{array}{c}
\delta \varepsilon_{11}^{h} \\
\delta \varepsilon_{22}^{h} \\
2 \delta \varepsilon_{12}^{h} \\
\delta \kappa_{11}^{h} \\
\delta \kappa_{22}^{h} \\
2 \delta \kappa_{12^{h}}^{h} \\
\delta \gamma_{1}^{h} \\
\delta \gamma_{2}^{h}
\end{array}\right]=\sum_{I=1}^{n_{e n}}\left[\begin{array}{cc}
N_{I, 1} \mathbf{x}_{, 1}^{h T} & \mathbf{0} \\
N_{I, 2} \mathbf{x}_{, 2}^{h T} & \mathbf{0} \\
N_{I, 1} \mathbf{x}_{, 2}^{h T}+N_{I, 2} \mathbf{x}_{, 1}^{h T} & \mathbf{0} \\
N_{I, 1} \mathbf{d}_{, 1}^{h T} & \hat{\mathbf{b}}_{11 I}^{h T} \\
N_{I, 2} \mathbf{d}_{, 2}^{h T} & \hat{\mathbf{b}}_{22 I}^{h T} \\
N_{I, 2} \mathbf{d}_{, 1}^{h T}+N_{I, 1} \mathbf{d}_{, 2}^{h T} & \hat{\mathbf{b}}_{12 I}^{h T}+\hat{\mathbf{b}}_{21 I}^{h T} \\
N_{I, 1} \mathbf{d}^{h T} & N_{I} \mathbf{b}_{I 1}^{h T} \\
N_{I, 2} \mathbf{d}^{h T} & N_{I} \mathbf{b}_{I 2}^{h T}
\end{array}\right]\left[\begin{array}{c}
\delta \mathbf{u}_{I} \\
\delta \boldsymbol{\beta}_{I}
\end{array}\right] } \\
\delta \boldsymbol{\varepsilon}^{h} & =\sum_{I=1}^{n_{e n}} \mathbf{B}_{I} \delta \mathbf{v}_{I}
\end{aligned}
$$


with $\mathbf{b}_{I \alpha}^{h}=\mathbf{T}_{M_{I}}^{T} \mathbf{x}_{, \alpha}^{h}$ and $\hat{\mathbf{b}}_{\alpha \beta I}^{h}=\hat{\mathbf{T}}_{I, \alpha}^{T} \mathbf{x}_{, \beta}^{h}$. The matrix $\hat{\mathbf{T}}_{I, \alpha}$ is defined by

$$
\hat{\mathbf{T}}_{I, \alpha}=\mathbf{T}_{, \alpha} \mathbf{M}_{I} N_{I}+\mathbf{T}\left(N_{I, \alpha} \mathbf{M}_{I}+N_{I} \mathbf{M}_{I, \alpha}\right) .
$$

5.3. Second variation of the potential

The finite element approximation of equation (24) reads

$$
\mathrm{L}[G(\mathbf{v}, \delta \mathbf{v})]=\bigcup_{e=1}^{n_{e l}} \sum_{I=1}^{n_{e n}} \sum_{K=1}^{n_{e n}}\left(\mathbf{f}_{I}^{e}+\mathbf{K}_{I K}^{e} \Delta \mathbf{v}_{K}\right)
$$

with the element stiffness matrix

$$
\mathbf{K}_{I K}^{e}=\int_{\Omega}\left(\mathbf{B}_{I}^{T} \mathbb{C} \mathbf{B}_{K}+\mathbf{G}_{I K}\right) \mathrm{d} \Omega
$$

and the residual vector

$$
\mathbf{f}_{I}^{e}=\int_{\Omega} \mathbf{B}_{I}^{T} \sigma \mathrm{d} \Omega-\mathbf{f}_{I}^{e, e x t} .
$$

The external loads are denoted by $\mathbf{f}_{I}^{e, e x t}$ and the initial stress stiffness matrix, which originates from the term $\Delta \delta \boldsymbol{\varepsilon}^{T} \boldsymbol{\sigma}$ in equation (25), by $\mathbf{G}_{I K}$. The factor $\Delta \delta \boldsymbol{\varepsilon}$ requires the second variation of the current orthogonal basis system. The interpolation of the linearized virtual strains

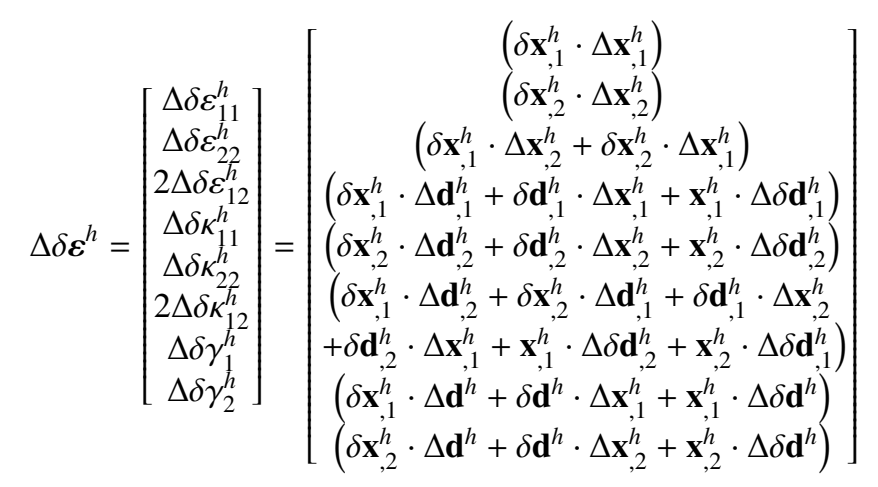

contains terms of the form $\mathbf{h} \cdot \Delta \delta \mathbf{d}^{h}$ and $\mathbf{h} \cdot \Delta \delta \mathbf{d}_{, \alpha}^{h}$, where $\mathbf{h}$ is an arbitrary vector. The derivation of these products can be found in Appendix A in detail. The resulting equations (A.1) and (A.14) are inserted into the interpolation of the linearized virtual strains (75). The membrane and shear part read

$$
\begin{array}{r}
\Delta \delta \varepsilon_{\alpha \beta}^{h}=\sum_{I=1}^{n_{e n}} \sum_{K=1}^{n_{e n}} \frac{1}{2}\left(N_{I, \alpha} N_{K, \beta}+N_{I, \beta} N_{K, \alpha}\right) \delta \mathbf{u}_{I}^{T} \mathbf{1} \Delta \mathbf{u}_{K} \\
\Delta \delta \gamma_{\alpha}^{h}=\sum_{I=1}^{n_{e n}} \sum_{K=1}^{n_{e n}}\left\{\delta \mathbf{u}_{I}^{T} N_{I, \alpha} N_{K} \mathbf{T}_{M_{K}} \Delta \boldsymbol{\beta}_{K}\right. \\
+\delta \boldsymbol{\beta}_{I}^{T} N_{I} N_{K, \alpha} \mathbf{T}_{M_{I}}^{T} \Delta \mathbf{u}_{K} \\
\left.+\delta \boldsymbol{\beta}_{I}^{T} N_{I} N_{K} \hat{\mathbf{M}}_{I K}\left(\mathbf{x}_{, \alpha}^{h}\right) \Delta \boldsymbol{\beta}_{K}\right\}
\end{array}
$$

whereas the curvatures

$$
\begin{aligned}
& \Delta \delta \kappa_{\alpha \beta}^{h}=\sum_{I=1}^{n_{e n}} \sum_{K=1}^{n_{e n}}\left\{\frac{1}{2} \delta \mathbf{u}_{I}^{T}\left[N_{I, \alpha} \hat{\mathbf{T}}_{K, \beta}+N_{I, \beta} \hat{\mathbf{T}}_{K, \alpha}\right] \Delta \boldsymbol{\beta}_{K}\right. \\
& +\frac{1}{2} \delta \boldsymbol{\beta}_{I}^{T}\left[N_{K, \beta} \hat{\mathbf{T}}_{I, \alpha}^{T}+N_{K, \alpha} \hat{\mathbf{T}}_{I, \beta}^{T}\right] \Delta \mathbf{u}_{K} \\
& +\frac{1}{2} \delta \boldsymbol{\beta}_{I}^{T}\left[\hat{\mathbf{M}}_{I K}^{L \alpha}\left(\mathbf{x}_{, \beta}^{h}\right)+\hat{\mathbf{M}}_{I K}^{R \alpha}\left(\mathbf{x}_{, \beta}^{h}\right)+\hat{\mathbf{M}}_{I K}^{L \beta}\left(\mathbf{x}_{, \alpha}^{h}\right)\right. \\
& +\hat{\mathbf{M}}_{I K}^{R \beta}\left(\mathbf{x}_{, \alpha}^{h}\right)+N_{I} N_{K} \hat{\mathbf{M}}_{I K, \alpha}\left(\mathbf{x}_{, \beta}^{h}\right) \\
& \left.\left.+N_{I} N_{K} \hat{\mathbf{M}}_{I K, \beta}\left(\mathbf{x}_{, \alpha}^{h}\right)\right] \Delta \boldsymbol{\beta}_{K}\right\}
\end{aligned}
$$


are more lengthy. All $\hat{\mathbf{M}}_{I K}$ matrices used in equations (77) and (76) are derived in Appendix A and provided by equations (A.2) and (A.15) . Finally the matrix $\mathbf{G}_{I K}$ is formed by computing the product

$$
\begin{aligned}
\Delta \delta \boldsymbol{\varepsilon}^{h T} \boldsymbol{\sigma} & =\sum_{I=1}^{n_{e n}} \sum_{K=1}^{n_{e n}} \delta \mathbf{v}_{I}^{T} \mathbf{G}_{I K} \Delta \mathbf{v}_{K} \\
& =\sum_{I=1}^{n_{e n}} \sum_{K=1}^{n_{e n}}\left[\begin{array}{c}
\delta \mathbf{u}_{I} \\
\boldsymbol{\beta}_{I}
\end{array}\right]^{T}\left[\begin{array}{cc}
\hat{n}_{I K} \mathbf{1} & \hat{\mathbf{m}}_{I K}^{u \beta}+\hat{\mathbf{q}}_{I K}^{u \beta} \\
\hat{\mathbf{m}}_{I K}^{\beta u}+\hat{\mathbf{q}}_{I K}^{\beta u} & \hat{\mathbf{M}}_{I K}(\hat{\mathbf{h}})+\hat{\mathbf{m}}_{I K}^{\beta \beta}
\end{array}\right]\left[\begin{array}{c}
\Delta \mathbf{u}_{K} \\
\Delta \boldsymbol{\beta}_{K}
\end{array}\right]
\end{aligned}
$$

with the following entries:

$$
\begin{aligned}
\hat{n}_{I K}= & n^{11} N_{I, 1} N_{K, 1}+n^{22} N_{I, 2} N_{K, 2}+n^{12}\left(N_{I, 1} N_{K, 2}+N_{I, 2} N_{K, 1}\right) \\
\hat{\mathbf{m}}_{I K}^{u \beta}= & m^{11} N_{I, 1} \hat{\mathbf{T}}_{K, 1}+m^{22} N_{I, 2} \hat{\mathbf{T}}_{K, 2}+m^{12}\left(N_{I, 1} \hat{\mathbf{T}}_{K, 2}+N_{I, 2} \hat{\mathbf{T}}_{K, 1}\right) \\
\hat{\mathbf{m}}_{I K}^{\beta u}= & m^{11} N_{K, 1} \hat{\mathbf{T}}_{I, 1}^{T}+m^{22} N_{K, 2} \hat{\mathbf{T}}_{I, 2}^{T}+m^{12}\left(N_{K, 1} \hat{\mathbf{T}}_{I, 2}^{T}+N_{k, 2} \hat{\mathbf{T}}_{I, 1}^{T}\right) \\
\hat{\mathbf{q}}_{I K}^{u \beta}= & \left(q^{1} N_{I, 1}+q^{2} N_{I, 2}\right) N_{K} \mathbf{T}_{M_{K}} \\
\hat{\mathbf{q}}_{I K}^{\beta u}= & \left(q^{1} N_{K, 1}+q^{2} N_{K, 2}\right) N_{I} \mathbf{T}_{M_{I}}^{T} \\
\hat{\mathbf{m}}_{I K}^{\beta \beta}= & \hat{\mathbf{M}}_{I K}^{L 1}\left(\mathbf{h}^{1}\right)+\hat{\mathbf{M}}_{I K}^{R 1}\left(\mathbf{h}^{1}\right)+\hat{\mathbf{M}}_{I K}^{L 2}\left(\mathbf{h}^{2}\right)+\hat{\mathbf{M}}_{I K}^{R 2}\left(\mathbf{h}^{2}\right) \\
& +\hat{\mathbf{M}}_{I K, 1}\left(N_{I} N_{K} \mathbf{h}^{1}\right)+\hat{\mathbf{M}}_{I K, 2}\left(N_{I} N_{K} \mathbf{h}^{2}\right)
\end{aligned}
$$

The vectors $\mathbf{h}$ read:

$$
\begin{aligned}
\hat{\mathbf{h}} & =q^{1} N_{I} N_{K} \mathbf{x}_{, 1}^{h}+q^{2} N_{I} N_{K} \mathbf{x}_{, 2}^{h} \\
\mathbf{h}^{1} & =m^{11} \mathbf{x}_{, 1}^{h}+m^{12} \mathbf{x}_{, 2}^{h} \\
\mathbf{h}^{2} & =m^{12} \mathbf{x}_{, 1}^{h}+m^{22} \mathbf{x}_{, 2}^{h}
\end{aligned}
$$

\section{Numerical examples}

The capability of the present shell formulation is demonstrated with the help of five numerical examples. The first two examples are taken from the shell obstacle course proposed in [10] and benchmark the accuracy of linear computations. The Scordelis-Lo roof, already briefly introduced in Section 4.1, compares the proposed approach for the computation of nodal basis systems and the interpolation of the director vector with a standard approach. With the help of the pinched cylinder the present shell formulation is compared to existing isogeometric shell formulations. Two further examples inquire the convergence behaviour in the nonlinear regime of the proposed formulation. The hemispherical shell with hole tests large rigid body rotations and large deformations. The cantilever subjected to end moment requires a proper treatment of large rotations. The last example demonstrates the improved accuracy of the present approach for free form surfaces with changing curvature. All computations use a St. Venant-Kirchhoff material model.

\subsection{Scordelis-Lo roof}

In Section 4.1 the Scordelis-Lo roof is used to demonstrate the interpolation error of the director for nodal basis systems attained by a closest point projection. The effect of this interpolation error in combination with the inaccurate interpolation of the variation of the director vector is shown in the following example. The standard geometry and material parameters are used. Length and radius are $L=50$ respective $R=25$ with a shell thickness $t=0.25$. The Young's modulus is $E=4.32 \cdot 10^{8}$ with a Poisson's ratio $v=0$. The system is loaded by a uniform vertical gravity load per unit area of $g=90$. Due to symmetry only one quarter of the system is modeled. In Fig. 3 the system is sketched, for details see [10]. Computations are linear. The vertical deformations in point A plotted in Fig. 3 converge to a value of $w=-0.3020247$. The computations with the present approach clearly show monotonously growing convergence behaviour for $h$-refinement for all three plotted orders of NURBS basis functions. In contrast to that, the standard approach overestimates the deformations for an order $p=6$. For an order $p=4$ the deformations are 


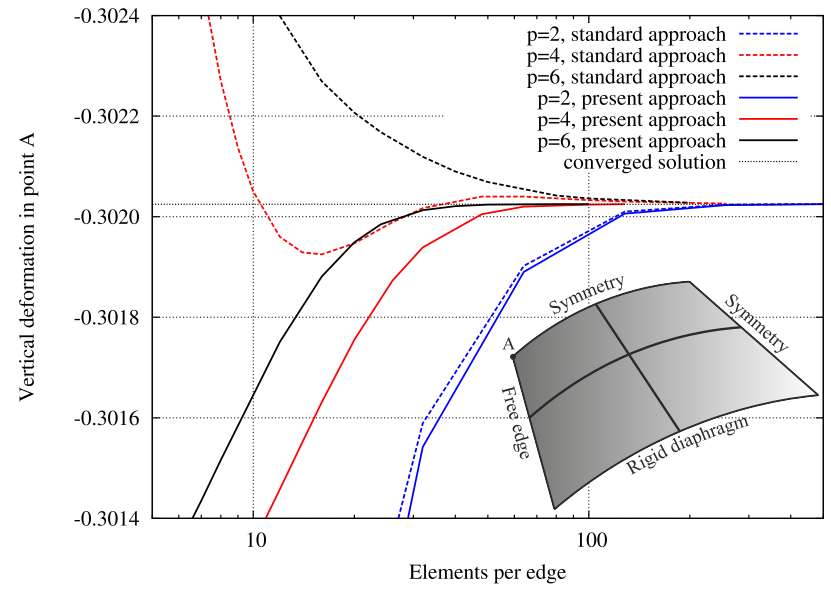

Figure 3: Scordelis-Lo roof: System sketch and comparison of deformations for $h$-refinement.

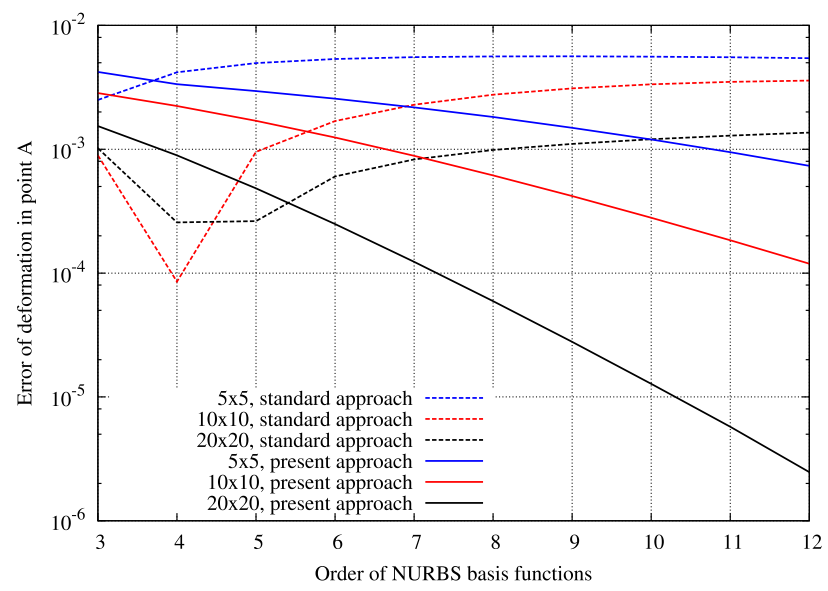

Figure 4: Scordelis-Lo roof: Deformation error for $k$-refinement.

overestimated for a coarse mesh, then show oscillatory behaviour and finally converge from above. For the lowest order possible $(p=2)$ only small differences occur.

To enlighten this issue a $k$-refinement study is provided in Fig. 4 . The chosen $k$-refinement strategy for increasing the order of the NURBS basis functions also elevates the continuity. For more details about refinement strategies see [16]. The deformation error plotted for both approaches for meshes with $5 \times 5,10 \times 10$ and $20 \times 20$ elements is calculated with the vertical deformation $w=-0.3020247$ in point A as converged solution. The deformation error for all three discretizations shows correct convergence behaviour for the present approach. The standard approach obviously diverges for rising orders. The quite low errors for an order $p=4$ are a result of the oscillatory behaviour visible in Fig. 3 and will not occur in general.

\subsection{Pinched cylinder}

The pinched cylinder is used to compare the accuracy of the present formulation with an isogeometric KirchhoffLove shell proposed by Kiendl et al. in [1] and the isogeometric Reissner-Mindlin shell of Benson et al. [4]. The system is sketched in Fig. 5 and consists of a cylinder which is constrained at both ends with a rigid diaphragm. The cylinder has a radius $R=300$ and a length $L=600$. The material is specified by a Young's modulus $E=3.0 \cdot 10^{6}$, a Poisson's ratio $v=0.3$ and a wall thickness $t=3$. More details can be found in [10]. Due to symmetry only one eigth of the system is modeled and sketched. The rigid diaphragm is considered as boundary condition. As the ratio between radius and wall thickness is $R / t=100$ the deformations entailed by shear deformation are negligible. 


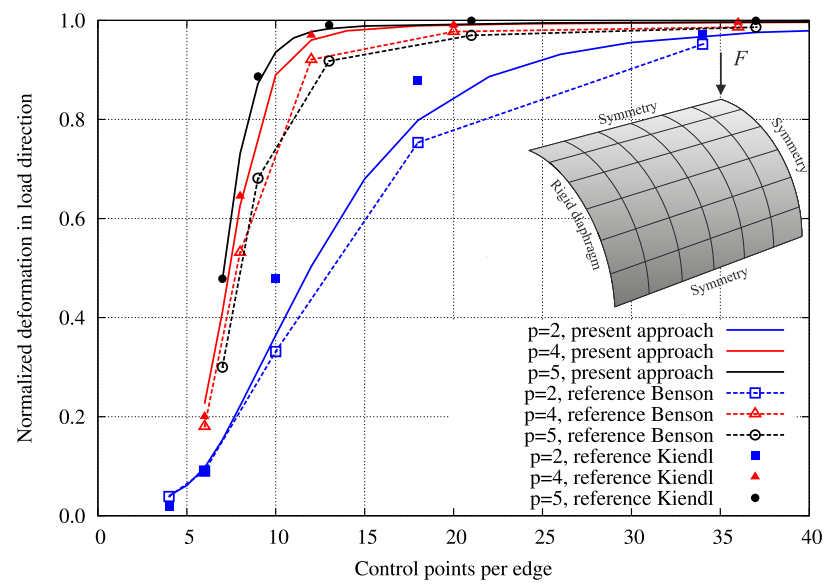

Figure 5: Pinched Cylinder: System sketch and comparison of deformations.

Thus, Reissner-Mindlin shell formulations should yield results comparable to Kirchhoff-Love shell formulations. All deformations plotted in Fig. 5 are normalized to $1.83 \cdot 10^{-5}$. Computations with the present approach coincide very well with the reference shell of Kiendl et al. [1] for orders $p=4$ and $p=5$. For the lowest order $p=2$ the presented Reissner-Mindlin shell clearly converges slower due to locking. The present formulation shows improved accuracy with rising order $p$ of the NURBS basis functions, which is not the case in the reference computations of Benson et al. [4].

\subsection{Hemispherical shell with hole}

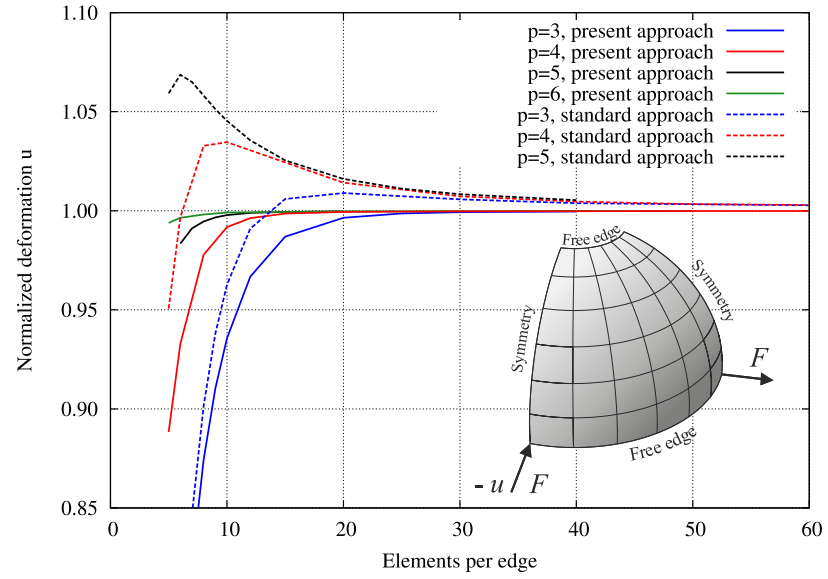

Figure 6: Hemispherical shell with hole: System sketch and comparison of deformations.

With the help of the hemispherical shell with hole the ability of the proposed shell formulation to handle large deformations and large rotations is demonstrated. No nonlinear reference results for isogeometric shell formulations are available at this time to the authors' knowledge. Thus, the present approach is compared to own computations with the standard approach. The system is sketched in Fig. 6. It is a hemispherical shell with an $18^{\circ}$ hole in the center. The upper and lower edge are free. The system is loaded with radial point loads $2 F$ on the equatorial edge with alternating orientation every $90^{\circ}$. The radius of the hemisphere is $R=10$ and the wall thickness is $t=0.04$. The St. Venant-Kirchhoff material has a Young's modulus $E=6.825 \cdot 10^{7}$ and a Poisson's ratio $v=0.3$. Due to symmetry only one fourth of the system is modeled. The load of $F=100$ is applied in ten loadsteps and yields large deformations and rotations. The converged deformation $u=-5.8614$ is used for the normalization of the deformations in Fig. 6 . It 
is clearly visible that the present approach yields convergence rates superior to those of the standard approach for all orders of basis functions and for all discretizations. The present approach with a polynomial order $p=6$ and $10 \times 10$ elements already causes a sufficient accurate solution with a deviation of $0.092 \%$, whereas the standard approach does not converge at all for this order, even for way smaller loadsteps and finer meshes.

\subsection{Cantilever subjected to end moment}

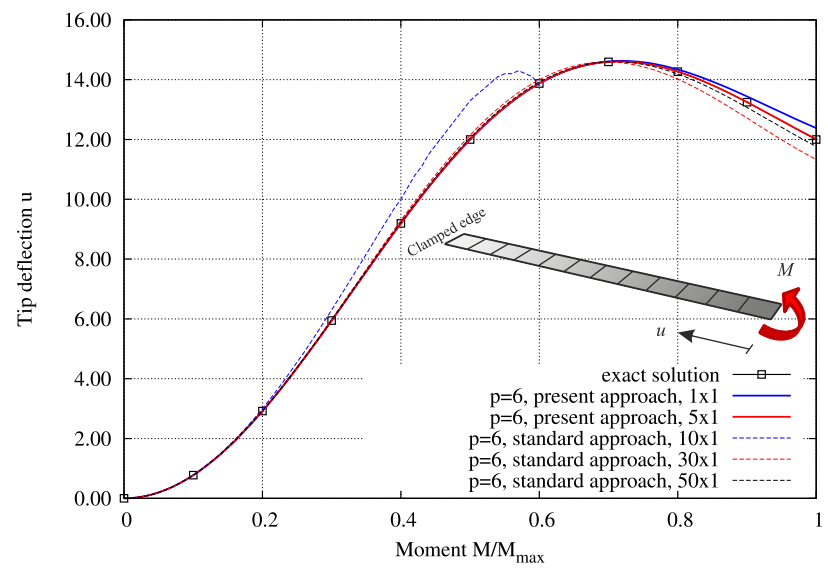

Figure 7: Cantilever subjected to end moment: System sketch and comparison of deformations.

The proper handling of large rotations is tested with a plane cantilever. One edge of the cantilever is clamped, whereas the other end is loaded with an end moment. The deformations of all control points in lateral direction are linked and the Poisson's ratio is set $v=0$ to entail a beam-like behaviour. The dimensions of the beam are $l=12$ and $b=1$ with a thickness $t=0.1$ and a Young's modulus $E=1.2 \cdot 10^{6}$. A sketch of the system is given in Fig. 7. An analytical solution for the displacement $u$ is given according to beam theory as

$$
u(M)=\left[\frac{M_{0}}{M} \sin \left(\frac{M}{M_{0}}\right)-1\right] l,
$$

where $M_{0}=E I / l=25 / 3$. The cantilever is fully rolled up for a moment $M_{\max }=2 \pi M_{0}$. The loading $M$ is applied in 10 loadsteps with $\Delta M=0.1 M_{\text {Max }}$ and should result in $u=12.0$ as deformation.

In Fig. 7 load-deflection curves are plotted for different discretizations with the standard and the present approach and compared to the exact solution. The standard approach with an order $p=6$ of the NURBS basis functions does not converge at all for an elementation with 10 elements in longitudinal direction. A fine mesh with $50 \times 1$ elements still entails a relatively large error of $1.9 \%$ for the standard approach. In contrast the proposed approach delivers superior results as it underestimates the deformation by $3.2 \%$ for $1 \times 1$ element and by $0.15 \%$ for $5 \times 1$ elements. The load-deflection curve for $5 \times 1$ elements with an order $p=6$ in Fig. 7 is indistinguishable from the exact solution. The results attained by the standard approach clearly degrade with rising order $p$ of the NURBS basis functions. Computations with the present approach require in general less load steps to attain convergent results than the standard approach. A discretization with $16 \times 1$ elements of order $p=4$ necessitates 10 load steps for the standard approach, whereas for the present approach 5 load steps suffice. In this example the computation of the nodal basis systems has no influence as the initial geometry is planar. Thus, the superior results of the present approach are merely due to the more accurate interpolation of the director vector.

\subsection{Double curved free form surface}

In contrast to the above standard benchmark examples the proposed new benchmark example has a changing curvature. Thus, the exact choice of the director vector and a proper treatment of the variation of the director vector is of huge importance. In Section 6.1 it is obvious that the proposed formulation yields superior results for the ScordelisLo roof, which features a constant curvature, but the differences are small which makes also the standard approach 


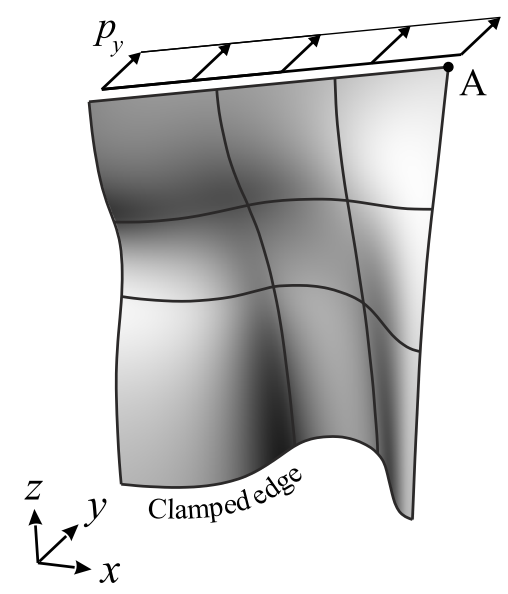

Figure 8: Double curved free form surface: System sketch.

useable for standard applications. The system presented in Fig. 8 is desigend to point out that the computation of free form surfaces requires the proper treatment of rotations. The information needed to construct the geometry in a modeling software is given in Appendix B. The lower edge is clamped and all other edges are free. On the top edge a line load of $p_{y}=10$ per unit length is applied in $y$-direction. The material is specified by a wall thickness $t=0.1$, a Young's modulus $E=1.2 \cdot 10^{6}$ and a Poisson's ratio $v=0.3$. The converged solution used for the deformation errors is $u_{y}=1.02786$. It is computed with $180 \times 180$ elements with a polynomial order $p=6$.

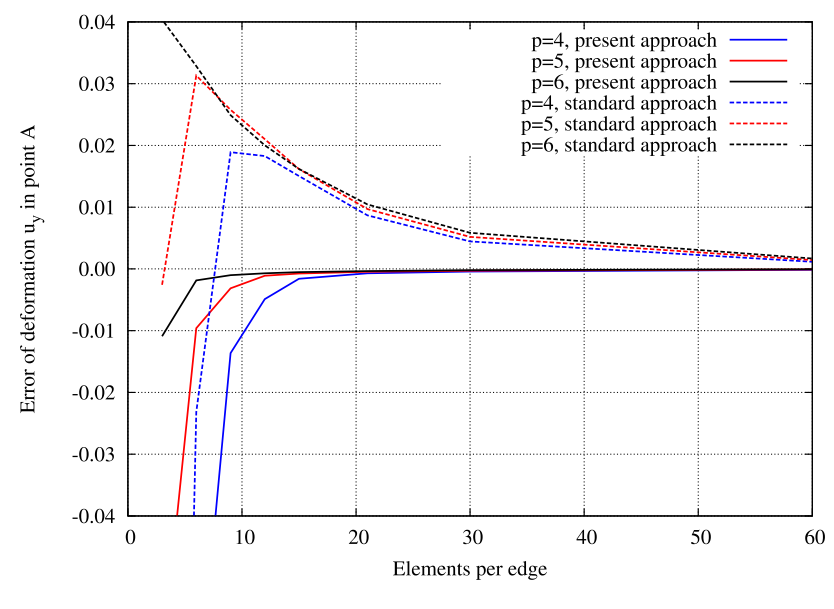

Figure 9: Double curved free form surface: Deformation error in point A.

The error of deformation plotted in Fig. 9 and 10 clearly shows the superior behavior of the proposed formulation. For meshes with $20 \times 20$ elements the standard approach yields an error of about $1 \%$, whereas all three orders plotted for the present approach deviate less than $0.1 \%$ from the converged solution. The convergence behavior for coarse meshes depicted in Fig. 9 shows that meshes with $10 \times 10$ elements and an order $p=5$ are sufficient for standard applications. In Fig. 10 a log-log graph is given to enlighten the convergence behavior for further mesh refinement. The error entailed by the present approach is always at least one magnitude smaller than that of the standard approach. Thus, the computation cost for a given error bound is much smaller for the present approach despite the much higher numerical effort per integration point for the present approach. In Tab. 1 an exemplary comparison is drawn. The predefined error bound is $\pm 0.1 \%$. Computations are performed on a 12 -core Intel $®$ Xeon ${ }^{\mathrm{TM}}$ machine. The assembly of the stiffness matrix is parallelized with OpenMP. The system is solved with the PARDISO solver from the Intel@ Math Kernel Library. 


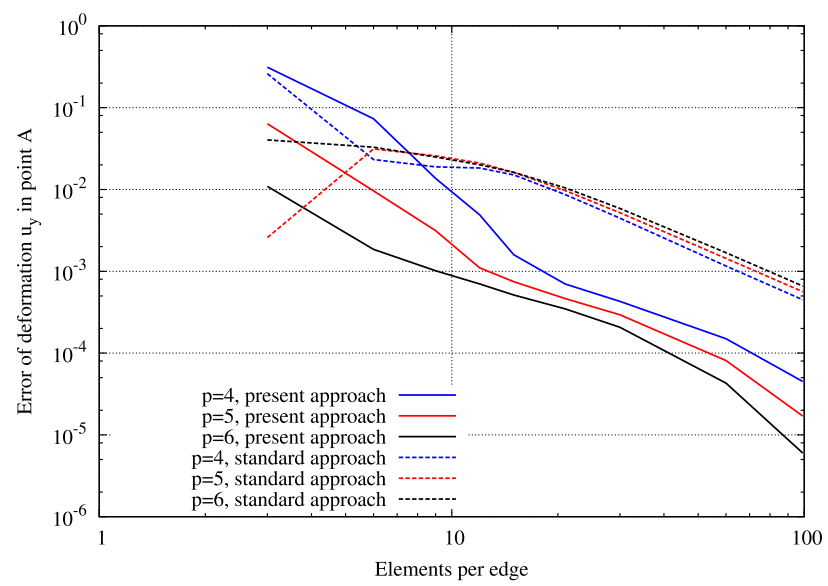

Figure 10: Double curved free form surface: Logscaled deformation error in point A.

\begin{tabular}{l||c|c|c|c|c} 
approach & elements & $\begin{array}{c}\text { integration } \\
\text { points }\end{array}$ & $\begin{array}{c}\text { time for } \\
\text { stiffness } \\
\text { matrix }\end{array}$ & $\begin{array}{c}\text { time for } \\
\text { solution }\end{array}$ & $\begin{array}{c}\text { error of } \\
\text { defor- } \\
\text { mation }\end{array}$ \\
\hline present, $p=4$ & 324 & 8100 & $0.8 \mathrm{~s}$ & $0.5 \mathrm{~s}$ & $-0.094 \%$ \\
standard, $p=4$ & 3969 & 99225 & $5.6 \mathrm{~s}$ & $3.9 \mathrm{~s}$ & $0.106 \%$ \\
present, $p=6$ & 81 & 3969 & $1.7 \mathrm{~s}$ & $0.5 \mathrm{~s}$ & $-0.102 \%$ \\
standard, $p=6$ & 6084 & 289116 & $58 \mathrm{~s}$ & $15.6 \mathrm{~s}$ & $0.104 \%$
\end{tabular}

Table 1: Comparison of computation cost.

\section{Conclusion}

An isogeometric Reissner-Mindlin shell formulation with correct convergence behaviour for $k$-refinement has been developed. The improved accuracy is due to the presented new method for the computation of nodal director vectors and basis systems and the more accurate interpolation of the current director vector and its variation. The main features of the proposed formulation are:

- The computation of exact nodal basis systems in the control points leads to interpolated director vectors, which are identical to the normal vector in the reference configuration. Thus, geometry and director vector are exact for every discretization.

- The current director vector is computed by an orthogonal rotation of the interpolated reference director vector. This entails an exact fulfillment of the inextensibility constraint.

- The proposed variation of the current director vector interpolating local rotations accounts for the discrepancy of interpolated basis systems and nodal basis systems. This results in more accurate results for curved structures and improved convergence behaviour for nonlinear computations. Especially for higher orders of NURBS basis functions larger load steps are possible than with standard formulations. Thus, computations with coarse meshes and high order are highly competitive.

The numerical results confirm these conclusions. The low number of integration points needed to attain precise results when high order basis functions are used makes the presented shell formulation potentially attractive for applications where high numerical effort per integration point occurs.

Future work will focus on the handling of complicated multi-patch structures with intersecting surfaces. 


\section{Acknowledgements}

This contribution was co-financed by the European Union from the European regional development fund (EFRE) and by the land Rhineland-Palatinate. The support is gratefully acknowledged.

\section{Appendix A. Second variation of the current director vector}

The derivation of $\mathbf{h}_{I} \cdot \Delta \delta \mathbf{d}_{I}$ conducted in [14] for nodal values $\mathbf{h}_{I}$ and $\Delta \delta \mathbf{d}_{I}$ can be adapted straight-forward for the term $\mathbf{h} \cdot \Delta \delta \mathbf{d}^{h}$. This results in

$$
\begin{aligned}
\mathbf{h} \cdot \Delta \delta \mathbf{d}^{h} & =\delta \mathbf{w} \cdot \mathbf{M}(\mathbf{h}) \Delta \mathbf{w} \\
& =\mathbf{H} \mathbf{T}_{3} \delta \boldsymbol{\beta} \cdot \mathbf{M}(\mathbf{h}) \mathbf{H T}_{3} \Delta \boldsymbol{\beta} \\
& =\mathbf{H} \mathbf{T}_{3}\left(\sum_{I=1}^{n_{e n}} N_{I} \mathbf{M}_{I} \delta \boldsymbol{\beta}_{I}\right) \cdot \mathbf{M}(\mathbf{h}) \mathbf{H} \mathbf{T}_{3}\left(\sum_{K=1}^{n_{e n}} N_{K} \mathbf{M}_{K} \delta \boldsymbol{\beta}_{K}\right) \\
& =\sum_{I=1}^{n_{e n}} \sum_{K=1}^{n_{e n}} N_{I} N_{K} \delta \boldsymbol{\beta}_{I}^{T} \hat{\mathbf{M}}_{I K}(\mathbf{h}) \Delta \boldsymbol{\beta}_{K}
\end{aligned}
$$

with

$$
\hat{\mathbf{M}}_{I K}(\mathbf{h})=\mathbf{M}_{I}^{T} \mathbf{T}_{3}^{T} \mathbf{H}^{T} \mathbf{M}(\mathbf{h}) \mathbf{H} \mathbf{T}_{3} \mathbf{M}_{I} .
$$

The Matrix $\mathbf{M}(\mathbf{h})$ is computed with $\mathbf{b}=\mathbf{d}^{h} \times \mathbf{h}$ by

$$
\begin{aligned}
\mathbf{M}(\mathbf{h}) & =\frac{1}{2}\left(\mathbf{d}^{h} \otimes \mathbf{h}+\mathbf{h} \otimes \mathbf{d}^{h}+\mathbf{t} \otimes \omega+\omega \otimes \mathbf{t}\right)+c_{10} \mathbf{1} \\
\mathbf{t} & =-c_{3} \mathbf{b}+c_{11}(\mathbf{b} \cdot \omega) \omega \\
c_{10} & =\bar{c}_{10}(\mathbf{b} \cdot \omega)-\left(\mathbf{d}^{h} \cdot \mathbf{h}\right) \\
c_{3} & =\frac{\omega \sin \omega+2(\cos \omega-1)}{\omega^{2}(\cos \omega-1)} \\
\bar{c}_{10} & =\frac{\sin \omega-\omega}{2 \omega(\cos \omega-1)} \\
c_{11} & =\frac{4(\cos \omega-1)+\omega^{2}+\omega \sin \omega}{2 \omega^{4}(\cos \omega-1)} .
\end{aligned}
$$

A series expansion of the coefficients is given in [14] to avoid numerical problems for small values of $\omega$.

The more complicated derivation of $\mathbf{h} \cdot \Delta \delta \mathbf{d}_{, \alpha}^{h}$ is attained by partial derivation of the first line in equation (A.1)

$$
\begin{aligned}
\left(\mathbf{h} \cdot \Delta \delta \mathbf{d}^{h}\right)_{, \alpha} & =(\delta \mathbf{w} \cdot \mathbf{M}(\mathbf{h}) \Delta \mathbf{w})_{, \alpha} \\
\mathbf{h} \cdot \Delta \delta \mathbf{d}_{, \alpha}^{h}+\mathbf{h}_{, \alpha} \cdot \Delta \delta \mathbf{d}^{h} & =(\delta \mathbf{w} \cdot \mathbf{M}(\mathbf{h}) \Delta \mathbf{w})_{, \alpha},
\end{aligned}
$$

which results after transposing in

$$
\begin{aligned}
\mathbf{h} \cdot \Delta \delta \mathbf{d}_{, \alpha}^{h}= & \delta \mathbf{w}_{, \alpha} \cdot \mathbf{M}(\mathbf{h}) \Delta \mathbf{w}+\delta \mathbf{w} \cdot \mathbf{M}(\mathbf{h}) \Delta \mathbf{w}_{, \alpha} \\
& +\delta \mathbf{w} \cdot \mathbf{M}_{, \alpha}(\mathbf{h}) \Delta \mathbf{w}-\delta \mathbf{w} \cdot \mathbf{M}\left(\mathbf{h}_{, \alpha}\right) \Delta \mathbf{w} .
\end{aligned}
$$

Equation (A.5) entails the need for the derivation of the quite involved matrix $\mathbf{M}(\mathbf{h})$. After lengthy derivations $\mathbf{M}_{, \alpha}(\mathbf{h})$ can be attained. The resulting matrix contains terms with the derivatives $\mathbf{h}_{, \alpha}$. They prove to cancel out with the matrix $\mathbf{M}\left(\mathbf{h}_{, \alpha}\right)$. Thus the last two terms on the right side of equation (A.5) are combined and the formulation reduces to

$$
\begin{aligned}
\mathbf{h} \cdot \Delta \delta \mathbf{d}_{, \alpha}^{h}= & \delta \mathbf{w}_{, \alpha} \cdot \mathbf{M}(\mathbf{h}) \Delta \mathbf{w}+\delta \mathbf{w} \cdot \mathbf{M}(\mathbf{h}) \Delta \mathbf{w}_{, \alpha} \\
& +\delta \mathbf{w} \cdot \overline{\mathbf{M}}_{, \alpha}(\mathbf{h}) \Delta \mathbf{w}
\end{aligned}
$$


with

$$
\begin{aligned}
\overline{\mathbf{M}}_{, \alpha}(\mathbf{h})= & \frac{1}{2}\left(\mathbf{d}_{, \alpha}^{h} \otimes \mathbf{h}+\mathbf{h} \otimes \mathbf{d}_{, \alpha}^{h}\right)+c_{10, \alpha} \mathbf{1} \\
& +\frac{1}{2}\left(+\overline{\mathbf{t}}_{, \alpha} \otimes \omega+\boldsymbol{\omega} \otimes \overline{\mathbf{t}}_{, \alpha}+\mathbf{t} \otimes \boldsymbol{\omega}_{, \alpha}+\boldsymbol{\omega}_{, \alpha} \otimes \mathbf{t}\right) \\
\overline{\mathbf{t}}_{, \alpha}= & -c_{3, \alpha} \mathbf{b}+-c_{3} \mathbf{b}_{, \alpha}+c_{11, \alpha}(\mathbf{b} \cdot \boldsymbol{\omega}) \omega \\
& +c_{11}\left(\mathbf{b}_{, \alpha} \cdot \boldsymbol{\omega}\right) \boldsymbol{\omega}+c_{11}\left(\mathbf{b} \cdot \boldsymbol{\omega}_{, \alpha}\right) \omega+c_{11}(\mathbf{b} \cdot \boldsymbol{\omega}) \boldsymbol{\omega}_{, \alpha} \\
\mathbf{b}_{, \alpha}= & \mathbf{d}_{, \alpha}^{h} \times \mathbf{h} \\
c_{10, \alpha}= & \bar{c}_{10, \alpha}(\mathbf{b} \cdot \boldsymbol{\omega})+\bar{c}_{10}\left(\mathbf{b}_{, \alpha} \cdot \boldsymbol{\omega}\right)+\bar{c}_{10}\left(\mathbf{b} \cdot \boldsymbol{\omega}_{, \alpha}\right)-\left(\mathbf{d}_{, \alpha}^{h} \cdot \mathbf{h}\right) .
\end{aligned}
$$

The required coefficients are

$$
c_{3, \alpha}=\frac{1}{180} \omega \omega_{, \alpha} \quad \bar{c}_{10}=\frac{1}{90} \omega \omega_{, \alpha} \quad c_{11}=\frac{-1}{3780} \omega \omega_{, \alpha}
$$

for $\omega \rightarrow 0$ and

$$
\begin{aligned}
c_{3, \alpha} & =\omega_{, \alpha} \frac{-\omega^{2}-\omega \sin \omega-4(\cos \omega-1)}{\omega^{3}(\cos \omega-1)} \\
\bar{c}_{10, \alpha} & =\frac{\omega_{, \alpha}}{2}\left(\frac{-\sin \omega-\omega}{\omega^{2}}-\frac{\sin \omega}{\cos \omega-1}\right) \\
c_{11, \alpha} & =\omega_{, \alpha} \frac{\cos \omega[-3 \omega(\sin \omega-\omega)-16(\cos \omega-2)]}{2 \omega^{5}(\cos \omega-1)^{2}} \\
& +\omega_{, \alpha} \frac{\omega \sin \omega\left(11-\omega^{2}\right)+3 \omega^{2}-16}{2 \omega^{5}(\cos \omega-1)^{2}}
\end{aligned}
$$

in other cases. With the two equations given in (57) $\delta \mathbf{w}$ can be expressed by

$$
\delta \mathbf{w}=\mathbf{H T}_{3} \delta \boldsymbol{\beta}
$$

and by derivation

$$
\delta \mathbf{w}_{, \alpha}=\mathbf{H}_{, \alpha} \mathbf{T}_{3} \delta \boldsymbol{\beta}+\mathbf{H T}_{3, \alpha} \delta \boldsymbol{\beta}+\mathbf{H T}_{3} \delta \boldsymbol{\beta}_{, \alpha}
$$

is attained. Here the operators $\delta$ and $\Delta$ are again interchangeable. This results in the interpolations

$$
\delta \mathbf{w}=\sum_{I=1}^{n_{e n}} N_{I} \mathbf{H} \mathbf{T}_{3} \mathbf{M}_{I} \delta \boldsymbol{\beta}_{I}
$$

and

$$
\delta \mathbf{w}_{, \alpha}=\sum_{I=1}^{n_{e n}}\left[N_{I}\left(\mathbf{H}_{, \alpha} \mathbf{T}_{3}+\mathbf{H T}_{3, \alpha}\right) \mathbf{M}_{I}+\right.
$$

$$
\left.\mathbf{H T}_{3}\left(N_{I, \alpha} \mathbf{M}_{I}+N_{I} \mathbf{M}_{I, \alpha}\right)\right] \delta \boldsymbol{\beta}_{I} .
$$

Insertion of $\delta \mathbf{w}, \Delta \mathbf{w}, \delta \mathbf{w}_{, \alpha}$ and $\Delta \mathbf{w}_{, \alpha}$ into equation (A.6) yields finally the expression

with

$$
\begin{aligned}
\mathbf{h} \cdot \Delta \delta \mathbf{d}_{, \alpha}^{h}=\sum_{I=1}^{n_{e n}} \sum_{K=1}^{n_{e n}} \delta \boldsymbol{\beta}_{I}^{T}\left[\hat{\mathbf{M}}_{I K}^{L \alpha}(\mathbf{h})+\hat{\mathbf{M}}_{I K}^{R \alpha}(\mathbf{h})\right. \\
\left.+N_{I} N_{K} \hat{\mathbf{M}}_{I K, \alpha}(\mathbf{h})\right] \Delta \boldsymbol{\beta}_{K}
\end{aligned}
$$

$$
\begin{aligned}
\hat{\mathbf{M}}_{I K, \alpha}(\mathbf{h}) & =\mathbf{T}_{H T M_{I}}^{T} \overline{\mathbf{M}}_{, \alpha}(\mathbf{h}) \mathbf{T}_{H T M_{K}} \\
\hat{\mathbf{M}}_{I K}^{L \alpha}(\mathbf{h}) & =\mathbf{T}_{H T M_{I, \alpha}}^{T} \mathbf{M}\left(N_{K} \mathbf{h}\right) \mathbf{T}_{H T M_{K}} \\
\hat{\mathbf{M}}_{I K}^{R \alpha}(\mathbf{h}) & =\mathbf{T}_{H T M_{I}}^{T} \mathbf{M}\left(N_{I} \mathbf{h}\right) \mathbf{T}_{H T M_{K, \alpha}} \\
\mathbf{T}_{H T M_{I, \alpha}} & =\left(\mathbf{H}_{, \alpha} \mathbf{T}_{3}+\mathbf{H} \mathbf{T}_{3, \alpha}\right) \mathbf{M}_{I} N_{I}+\mathbf{H} \mathbf{T}_{3}\left(N_{I, \alpha} \mathbf{M}_{I}+N_{I} \mathbf{M}_{I, \alpha}\right) \\
\mathbf{T}_{H T M_{I}} & =\mathbf{H T}_{3} \mathbf{M}_{I}
\end{aligned}
$$




\section{Appendix B. Geometry of the double curved free form surface}

The double curved free form surface used in Section 6.5 can be constructed uniquely from four boundary B-Spline curves. In Tab. B. 2 the control points for all four boundary curves are given. As $w=1$ holds for all control points a B-Spline surface is formed. The knot vector

$$
\boldsymbol{\Xi}=\left[0,0,0,0, \frac{1}{3}, \frac{2}{3}, 1,1,1,1\right]
$$

is identical for all curves. The order of the B-Spline basis functions is $p=3$ and the number of control points is $n=6$. Thus, the coarsest mesh consists of $3 \times 3$ elements.

\begin{tabular}{l|c|c|c|c} 
& top & bottom & left & right \\
\hline Control points & $0,0,15$ & $0,0,0$ & $0,0,0$ & $11,0,0$ \\
$(\mathrm{x}, \mathrm{y}, \mathrm{z})$ & $\frac{11}{9}, \frac{2}{3}, 15$ & $5,0,0$ & $0,0,5$ & $11,0, \frac{8}{3}$ \\
& $\frac{11}{3}, 2,15$ & $5,5,0$ & $0,2,7$ & $11, \frac{2}{\frac{62}{9}}$ \\
& $\frac{22}{3}, 4,15$ & $10,5,0$ & $0,2,10$ & $11, \frac{17}{9}, \frac{101}{9}$ \\
& $\frac{88}{9}, \frac{16}{3}, 15$ & $10,0,0$ & $0,0,12$ & $11, \frac{13}{3}, \frac{41}{3}$ \\
& $11,6,15$ & $11,0,0$ & $0,0,15$ & $11,6,15$
\end{tabular}

Table B.2: Control points of the four boundary curves.

\section{References}

[1] J. Kiendl, K.-U. Bletzinger, J. Linhard, R. Wüchner, Isogeometric shell analysis with Kirchhoff-Love elements, Comput. Meth. Appl. Mech. Engrg. 198 (2009) 3902-3914.

[2] J. Kiendl, Y. Bazilevs, M.-C. Hsu, R. Wüchner, K.-U. Bletzinger, The bending strip method for isogeometric analysis of Kirchhoff-Love shell structures comprised of multiple patches, Comput. Meth. Appl. Mech. Engrg. 199 (2010) 2403-2416.

[3] R. Echter, M. Bischoff, Numerical efficiency, locking and unlocking of NURBS finite elements, Comput. Meth. Appl. Mech. Engrg. 199 (2010) 374-382.

[4] D. J. Benson, Y. Bazilevs, M.-C. Hsu, T. J. R. Hughes, Isogeometric shell analysis: The Reissner-Mindlin shell, Comput. Meth. Appl. Mech. Engrg. 199 (2010) 276-289.

[5] D. J. Benson, Y. Bazilevs, M.-C. Hsu, T. J. R. Hughes, A large deformation, rotation-free, isogeometric shell, Comput. Meth. Appl. Mech. Engrg. 200 (2011) 1367-1378.

[6] J. C. Simo, D. D. Fox, On a stress resultant geometrically exact shell model. part I: Formulation and optimal parametrization, Comput. Meth. Appl. Mech. Engrg. 72 (1989) 267-304.

[7] L. Piegl, W. Tiller, The NURBS book, Monographs in visual communications, Springer, Berlin, second edition, 1997.

[8] J. A. Cottrell, T. J. R. Hughes, Y. Bazilevs, Isogeometric analysis: Toward integration of CAD and FEA, Wiley, Chichester, 2009.

[9] S. Klinkel, F. Gruttmann, W. Wagner, A mixed shell formulation accounting for thickness strains and finite strain 3D material models, Int. J. Num. Meth. Engng 74 (2008) 945-970.

[10] T. Belytschko, H. Stolarski, W. K. Liu, N. Carpenter, J. S.-J. Ong, Stress projection for membrane and shear locking in shell finite elements, Comput. Meth. Appl. Mech. Engrg. 51 (1985) 221-258.

[11] C. L. Lawson, R. J. Hanson, Solving least squares problems, volume 15 of Classics in applied mathematics, SIAM, Philadelphia, 1995.

[12] F. Gruttmann, W. Wagner, Structural analysis of composite laminates using a mixed hybrid shell element, Comput. Mech. 37 (2006) $479-497$.

[13] T. J. R. Hughes, The finite element method: Linear static and dynamic finite element analysis, Dover Publ., Mineola, NY, reprint. edition, 2000.

[14] F. Gruttmann, R. Sauer, W. Wagner, Theory and numerics of three-dimensional beams with elastoplastic material behaviour, Int. J. Num. Meth. Engng 48 (2000) 1675-1702.

[15] J. C. Simo, D. D. Fox, M. S. Rifai, On a stress resultant geometrically exact shell model. part III: Computational aspects of the nonlinear theory, Comput. Meth. Appl. Mech. Engrg. 79 (1990) 21-70.

[16] T. J. R. Hughes, J. A. Cottrell, Y. Bazilevs, Isogeometric analysis: CAD, finite elements, NURBS, exact geometry and mesh refinement, Comput. Meth. Appl. Mech. Engrg. 194 (2005) 4135-4195. 